\title{
A Thermodynamic Theory for Reacting Mixtures in Electromagnetic Fields (*).
}

\author{
RINaldo BoRghesanI (Genova) (**) (***)
}

A Beniamino SEGRE nel suo $70^{\mathrm{mo}}$ compleanno

Sunto. - In questo lavoro l'Autore formula una teoria termodinamica per una miscela costituita di materiali rigidi stazionari, che non diffondono ma che possono reagire chimicamente tra di loro e che sono inoltre soggetti all'azione di campi elettromagnetici.

Summary. - In this paper the Author gives a self consistent thermodynamic description of a mixture of rigid stationary materials. In this analysis the diffusion effects are neglected whyle the chemical reactions are taken into account; moreover the influence on the mixture due to the presence of electromagnetic fields is discussed in detail.

\section{1. - Introduction.}

It is well known that much of the recent work on mixture theories (i.e. mechanical and thermomechanical theories of materials consisting of several constituents with the possibility of mass diffusion and chemical reactions) has been based upon the equations of balance given by C. A. TrUESDELL [1], [2], [4], [13]. In this connection a wide extension of Truesdell's methods, so as to include also electromagnetic phenomena, was proposed by P. D. KeLLY in ref. [9].

Relevant contributions to the rational analysis of reacting mixtures of elastic and of viscoelastic materials (both in the absence of diffusion and in the general case when diffusion effects cannot be omitted) were given by R. M. BowEN in a series of papers [12], [16], [17], [19], by R. M. BowEN and J. C. Wrese in ref. [20], and by R. M. BowEN and D. J. Garcia in ref. [21]. Further developements in the description of reacting mixtures of fluids were recently proposed by M. E. GURTIN and A. S. VARGas [22] and by M. E. GuRTin [23].

In my opinion, one of the basic ideas in the mathematical theory of reacting materials of any type is that the local chemical effects can be examined by assigning a suitably defined "internal" vector field (called extent of reaction) and by assuming that each thermodynamical quantity must functionally depend also on this vector field. In this sense, as remarked by R. M. BowEN [14], we can adopt an "internal

$\left(^{*}\right)$ Some parts of this paper were read at the italo-american meeting on "Mixtures and Structured Continua" organized by proff. G. F. CAPRIz and C. A. Trutesdexi (C.I.S.M, Udine, Italy; 17-22 June 1974), see [30]. This work was supported by the "Gruppo Nazionale per la Fisica Matematica " of C.N.R.

(**) Istituto Matematico dell'Università di Genova (Italy).

(***) Entrata in Redazione il 25 luglio 1974.

20 - Annali di Matematica 
variables formalism " as the one developed by CoLEMAN and GURTIN [11], taking also into account the deeper chemical interpretations given by R. ARIS [10], [15] and by R. M. BowEN [16] (1).

On the other side, B. D. Coleman and E. H. DHL laid down the foundations of a self consistent thermodynamical theory of electromagnetic fields in stationary rigid materials with memory [24], [25], whyle R. BoRGHESANI adopted the concept of "quasi response" in order to introduce an analogous, though not completely equivalent, description of the same subject [26]. Obviously, when memory effects are neglected, the conclusions of the two approaches must be identical; and this is actually the case.

The present work is concerned with a thermodynamical analysis of a mixture consisting of stationary rigid materials, where chemical reactions are present and diffusion is omitted, with the fundamental assumption of an instantaneous electromagnetic response. This analysis should be regarded as a first step towards a consistent mathematical formulation of the so called "polarized solid-state reactions" within the framework of Continum Thermomechanics.

The main results of our study may be summarized as follows:

a) the extent of reaction vector must belong to the "restricted reaction space", which modifies the "reaction space" introduced by $R$. ARIS in ref. [10] (see sects. 2,3$)$;

b) the relations deduced by B. D. CoLEMAN and H. E. DHL for a simple stationary rigid material are generalized to the mixture in exam in the most natural way (see sects. 4,5 );

c) the definitions of "equilibrium", of "thermal equilibrium " and of "classical equilibrium " are introduced and their mutual relations discussed in detail (sect. 6);

d) some new expressions for the heat flux and the electric current "near a classical equilibrium state in a "polarized" reacting mixture are derived and commented (sect. 7);

e) finally, as far as non-conducting mixtures are concerned, an interesting connection between the classie "mass aetion law" and our general framework is described (sect. 8).

\section{2. - Basic definitions and assumptions. Mass and charge balance laws.}

In this section we give a short account of some definitions and assumptions, which are commonly used in the classical theory of mixtures. Obviously our survey

(1) An interesting account of the meaningful results obtained by R. M. BowEN is briefly outlined in the 9-th lecture of Truesdell's book on Rational Thermodynamics, Mc Graw-Hill, 1969, see ref. [18]. 
lacks completeness and we refer to the basic papers, quoted in the Introduction, for an exhaustive description.

Let us consider a mixture of $n$ chemically reacting constituent bodies $\mathfrak{B}_{a}(a=1,2, \ldots, n)$, where each body is a set with the well known structure prescribed by W. NoLL [3].

We define a configuration of the body $\mathfrak{B}_{a}$ as a homeomorphism $\chi_{a}$ of $\mathfrak{B}_{a}$ onto a region of the three-dimensional Euclidean space $\varepsilon$; whyle a motion of the body $\mathcal{B}_{a}$ is a one-parameter family of configurations $\chi_{a \mid t}$ where $t \in(-\infty, \infty)$ is the time. Then the position of the particle $X_{a}$ of $\mathfrak{B}_{a}$ at the time $t$ is

$$
x=\chi_{a] t}\left(X_{a}\right)=\chi_{a}\left(X_{a}, t\right)
$$

where $\chi_{a}: \Re_{a} \times(-\infty, \infty) \rightarrow \mathcal{E}$ is assumed to be invertible for each $t$. In a very general way, we can say that the region, occupied by $\mathcal{H}_{\alpha}$ at the time $t$ in the Euclidean space $\mathcal{E}$, is a compact set, with piecewise smooth boundaries, given by

$$
\chi_{a}\left(\mathcal{B}_{a}, t\right)=\left\{\chi_{a}\left(X_{a}, t\right): X_{a} \in \mathscr{B}_{a}\right\}
$$

Consequently, the mixture at the time $t$, denoted by $\mathcal{B}_{t}$, is so defined

$$
\mathscr{B}_{t}=\bigcup_{a \in N}\left\{X_{a}: \chi_{a}\left(X_{a}, t\right) \in \bigcap_{a \in N} \chi_{a}\left(\mathscr{B}_{a}, t\right)\right\}
$$

where $N$ is the set of all positive integers $a$ such that $1 \leqslant a \leqslant n$. From defs. (2.1) and (2.3) we infer that each point $x \in \bigcap_{a \in N} \boldsymbol{\chi}_{a}\left(\Re_{a}, t\right)$ is occupied by exactly $n$ particles, one for each body $\mathfrak{B}_{a}$. Obviously the part of $\mathfrak{B}_{a}$ that is in the mixture at the time $t$ is clearly given by $\mathfrak{B}_{a} \cap \mathfrak{B}_{t}$.

A reference configuration for the body $\mathfrak{乃}_{a}$ is a fixed configuration $\boldsymbol{K}_{a}: \mathfrak{B}_{a} \rightarrow \mathcal{\delta}$. Denoting by $\boldsymbol{X}_{a}=\boldsymbol{K}_{a}\left(X_{a}\right)$ the position occupied by $X_{a}$ in the reference configuration, from (2.1) it follows that

$$
\boldsymbol{x}=\boldsymbol{\chi}_{a}\left[\boldsymbol{K}_{a}^{-1}\left(\boldsymbol{X}_{a}\right), t\right]=\left(\boldsymbol{\chi}_{a}\right)_{\mathbf{K}}\left(\boldsymbol{X}_{a}, t\right)
$$

where the "deformation " function $\left(\chi_{a}\right)_{\mathbf{K}}$ and its inverse $\left(\chi_{a}\right)_{\mathbf{K}}^{-1}$ are usually assumed as $\mathrm{C}^{2}$ functions of their arguments.

By adopting the reference configurations, we can give definitions analogous to defs. (2.2) and (2.3). More precisely, the region oceupied by $\mathfrak{B}_{a}$ in its reference configuration is

$$
\boldsymbol{K}_{a}\left(\mathfrak{B}_{a}\right)=\left\{\boldsymbol{K}_{a}\left(X_{a}\right): X_{a} \in \mathfrak{B}_{a}\right\}
$$


whyle the mixture, denoted now by $\mathfrak{B}$, is to be regarded as

$$
\mathfrak{B}=\bigcup_{a \in \mathcal{N}}\left\{X_{a}: \boldsymbol{K}_{a}\left(X_{a}\right) \in \bigcap_{a \in N} \boldsymbol{K}_{a}\left(\mathfrak{B}_{a}\right)\right\} .
$$

In order to avoid the difficulties which arise when one attempts to consider interaetions between material defermations and motions and the electromagnetic fields, we consider only rigid stationary constituent bodies; in this case, the definitions (2.2) and (2.3) become essentially the same as those expressed by (2.5) and (2.6).

Our further assumption is the absence of diffusion among the constituent bodies, which allows us to write

$$
\boldsymbol{K}_{1}\left(\mathfrak{B}_{1}\right)=\boldsymbol{K}_{2}\left(\mathfrak{B}_{2}\right)=\ldots=\boldsymbol{K}_{\mathfrak{r}}\left(\mathfrak{B}_{n}\right)
$$

Since we confine our attention to rigid materials $\mathfrak{B}_{a}$, we may identify them with the regions $\mathcal{R}_{a}$ they occupy in the Euclidean space $\mathcal{E}$, for there is no reason to distinguish a particle $X_{a} \in \mathcal{B}_{a}$ from its position $\boldsymbol{x} \in \mathcal{E}$; then eqs. (2.7) read

$$
\mathfrak{R}_{1}=\mathfrak{R}_{2}=\ldots=\mathfrak{R}_{n} .
$$

Thus our mixture of rigid stationary constituent bodies without diffusion can be conveniently represented by the common region of the Euclidean space in eqs. (2.7) or in eqs. (2.8); namely

$$
R=\left\{x: x \in \bigcap_{a \in N} R_{a}\right\}
$$

As a result of the previous discussion, all the scalar, vector or tensor valued functions-describing any property whatsoever of the mixture-must be defined on $\mathcal{R} \times(-\infty, \infty)$.

Now, if we denote with $\varrho_{a}$ the mass density of the constituent body $\mathcal{B}_{a}$ at $(x, t) \in \mathcal{R} \times(-\infty, \infty)$, then the mass density of the mixture at the same point and at the same time is

$$
\varrho=\sum_{1}^{n} \varrho_{a}
$$

If we introduce the mass concentration $e_{a}=\varrho_{a} l \varrho$ of $\mathfrak{B}_{a}$ at $(x, t) \in \Re \times(-\infty, \infty)$, eq. (2.10) yields

$$
\sum_{1}^{n} c_{a}=1
$$

In our case, the balance of mass for the $a$-th constituent reads [1], [2], [14]

$$
\varrho c_{a}=\dot{\varrho}_{a} \quad a=1,2, \ldots, n,
$$

where $t_{a}$ is the so called "mass growth» of $\mathfrak{S}_{a}$. 
It is a matter of fact that, when chemical reactions are taken into account, the balance law (2.12) fails to reflect all that can be said regarding the conservation of mass in a mixture. Obviously this insufficienty is due to the atomic and molecular character of all chemical phenomena which, consequently, lie outside the classical framework of Continuum Thermomechanics. In order to overcome such a difficulty we need a type of balance law that ensures a mass conservation also at the atomic level, as the well known "law of permanence of the atomic substances " of chemical kinetics.

More precisely, if we denote with the positive number $A^{b}(b=1,2, \ldots, v)$ the "atomic weight" of the $b$-th atomic substance, then the "molecular weight " of the $a$-th constituent body $\mathfrak{B}_{a}(a=1,2, \ldots, n)$ is a positive number given by

$$
M^{a}=\sum_{1}^{v} S_{b}^{a} A^{b} \quad a=1,2, \ldots, n
$$

In eq. $(2,13)$ the positive integers $S_{b}^{a}$, called stoichiometric coefficients, represent the specific mass of the $b$-th atomic substance in the $a$-th consituent body; the rank of the matrix $\left\|S_{b}^{a}\right\|$ is a positive integer $\sigma$ such that $\sigma \leqslant \min (n, v)$.

Thus, in most physical-chemical circumstances, it is permissible to assert the conservation of mass for every atomio substance making up each constituent body; this axiom takes the form [1], [2], [4], [16], [17]

$$
\sum_{1}^{n_{B}} a S_{b}^{a} \frac{\stackrel{+}{c}_{a}}{M^{a}}=0 \quad b=1,2, \ldots, v .
$$

Since our analysis is concerned with chemical reactions which occur at the presence of electromagnetic fields, it is necessary to assume also the conservation of charge for every atomic substance in each constituent body; namely

$$
\sum_{1}^{n} S_{b}^{a} \frac{Q^{a} c_{a}^{+}}{M^{a}}=0 \quad b=1,2, \ldots, v
$$

where $Q^{a} \neq 0$ is the specific charge density of the $a$-th constituent, with $Q^{a} \neq Q^{a^{\prime}}$ $\forall a \neq a^{\prime}=1,2, \ldots, n^{(1)(2)}$. Taking eq. (2.13) and eq. (2.14) into account, we find easily

$$
\sum_{1}^{n}{ }_{a}^{+} c_{a}=0
$$

(1) Notice that, here and in the sequel, the specific charge density is a specific density of "free " charge in the sense that the charge density of the $a$ th constituent is $k_{\alpha}=\varrho_{a} Q^{a}$ when the electric polarization vector $\boldsymbol{P}_{a}$ is neglected.

$\left.{ }^{2}\right)$ It is obvious that an axiom of the type (2.15) deserves a deep interest in every question related to the dynamies of ionized gases and plasms-see, e.g., S. I. PAI, Magnetogas- 
which eusures that the net supply of mass to the mixture vanishes. The last equation implies obviously the constancy of the mass density for the whole mixture, i.e.

$$
\varrho=\varrho_{0}=\text { const }
$$

In a similar way, from eq. (2.13) and (2.15) we can derive the conservation of the charge for the mixture, namely

$$
\sum_{x}^{n} Q^{a} \hat{\boldsymbol{c}}_{a}^{+}=0^{(3)}
$$

If we introduce now the "molar concentration " $w_{a}=c_{a} / M^{\alpha}$ and the "molar growth" $\stackrel{+}{w}_{a}=\stackrel{+}{c}_{a} / M^{a}$, the balance of mass for each constituent togethet with the mass conservation of the whole mixture yield

$$
\dot{w}_{a}=\stackrel{+}{w}_{a} \quad a=1,2, \ldots, n .
$$

Moreover, the following relations

$$
\sum_{1}^{n} M^{a} w_{a}=1
$$

and

$$
\sum_{1}^{n} S_{b}^{a} \stackrel{+}{w}_{a}=0, \quad \sum_{1}^{n} Q^{a} S_{b}^{a} \dot{\dot{w}}_{a}=0, \quad b=1,2, \ldots, \nu
$$

must hold as a direct consequence of def. (2.11) and of the two axioms (2.14), (2.15).

\section{3. - Constituent space and restricted reaction subspace. Extent of reaction vector.}

In order to give a more compact form to some equations of the previous section, it is convenient to introduce an $n$-dimensional linear inner product space $R^{n}$, called

dynamics and Plasms Dynamics, Springer-Verlag (1962)-. As far as the Author is aware, the axiom (2.15) makes its first appearance in the periodical literature on the modern theory of mixtures in the paper of P. D. KELIY [9]. During a conversation held in Pisa in February 1974, professor C. A. Truespect has kindly shown to me the proofs of a forthcoming paper by R. BEXACH and I. MULLER [29], where the axiom (2.15) is taken into account.

Anyhow, both in ref. [9] and in ref. [29], the implications of the axiom within the framework of Bowen's formalism [16] are not explored.

${ }^{(3)}$ Obviously eq. (2.18) does not imply an analogous conservation law for the density $k_{a}$ $(a=1,2, \ldots, n)$, because this conservation must heavily depend on Maxwell field equations for the constituent $\mathfrak{B}_{a}$. 
a constituent space by R. M. BowEN [16] ( $\left.{ }^{1}\right)$. If $B \equiv\left\{e_{i}: i=1,2, \ldots, n\right\}$ is a basis of $R^{n}$ and $B^{-1} \equiv\left\{\boldsymbol{e}^{j}: j=1,2, \ldots, n\right\}$ is its reciprocal basis, then we can define the "concentration vector" and the "reaction vector" in the following way

$$
\boldsymbol{w}=\sum_{1}^{n} w_{a} \boldsymbol{e}^{a} \quad \text { and } \quad \stackrel{+}{\boldsymbol{w}}=\sum_{1}^{n} \stackrel{+}{w}_{a} \boldsymbol{e}^{a}
$$

respectively.

According to def. (3.1) $)_{2}$, the first of eqs. (2.21) becomes

$$
\stackrel{+}{\boldsymbol{w}} * \boldsymbol{S}_{b}=0 \quad b=1,2, \ldots, y,
$$

where $S_{b}=\sum_{1}^{n} S_{b}^{a} e_{a}$ and «*» denotes the inner product in the constituent space (2).

Since the rank of the matrix $\left\|S_{b}^{a}\right\|$ is the positive integer $\sigma \leqslant \min (n, v)$, we can select $\sigma$ of the vectors $S_{b}$ to be linearly indipendent. These vectors generate a linear subspace of the constituent space and such a subspace is the orthogonal complement of the so-called reaction subspace $\mathrm{S}$, introduced by $\mathrm{R}$. ARIS [10], [16]; namely we have

$$
\mathcal{S}^{\perp} \equiv L\left\{\boldsymbol{S}_{b}: b=1,2, \ldots, \sigma\right\}, \quad \operatorname{dim} \mathcal{S}^{\perp}=\sigma,
$$

and

$$
\mathcal{S} \equiv\left\{\boldsymbol{y} \in \boldsymbol{R}^{n}: \boldsymbol{y} * \boldsymbol{S}_{b}=\mathbf{0} ; b=1,2, \ldots, \sigma\right\}, \quad \operatorname{dim} \mathrm{S}=n-\sigma,
$$

where $R^{n}=\$ \oplus S^{\perp},\left(S^{\perp}\right)^{\perp}=S$. Then the condition (3.2) and the definition (3.4) yield the following result due to R. M. BowEN:

THEOREM 1. - The mass of the atomic substances of a reacting mixture is conserved if and only if the reaction vector lies in the $n-\sigma$-dimensional reaction subspace of the constituent space; i.e.

$$
\stackrel{+}{\boldsymbol{w}} \in \mathrm{S} \subset R^{n}
$$

If we recall again def. $(3.1)_{2}$, the second of eqs. (2.21) takes the form

$$
\stackrel{+}{\boldsymbol{w}} * T_{b}=0 \quad b=1,2, \ldots, \nu .
$$

(1) The inner product is completely arbitrary and has no physical meaning; its introduction is only due to formal convenience.

$\left(^{2}\right)$ As it is well known, the relation between the vectors of $B$ and the vectors of $B^{-1}$ is

$$
\boldsymbol{e}^{j}=\sum_{1}^{n} G_{i}^{j} e_{i} \quad j=1,2, \ldots, n,
$$

where $G_{i}^{i}$ is the reciprocal element of $g_{i j}$ in the square matrix

$$
\left\|g_{i j} \equiv \boldsymbol{e}_{i} * \boldsymbol{e}_{j}: i, j=1,2, \ldots, n\right\| \text {. }
$$


In eq. (3.6) we set $\boldsymbol{T}_{b}=\sum_{1}^{n} T_{b}^{a} \boldsymbol{e}_{a}=\sum_{1}^{n} Q^{a} S_{b}^{a} \boldsymbol{e}_{a}$ with the obvious observation that the ranks of the two matrices $\left\|T_{b}^{a}\right\|$ and $\left\|S_{b}^{a}\right\|$ are identical. Following the same procedure as before, we can introduce a linear subspace of the constituent space which is generated by $\sigma$ linearly indipendent vectors $\boldsymbol{T}_{b}$ and which is the orthogonal complement of the so called "charge subspace" $\mathfrak{C}$. Then we can write

$$
\mathfrak{G}^{\perp} \equiv L\left\{R_{b}: b=1,2, \ldots, \sigma\right\}, \quad \operatorname{dim} \mathfrak{G}^{\perp}=\sigma,
$$

and

$$
\mathfrak{G} \equiv\left\{\boldsymbol{y} \in R^{n}: \boldsymbol{y} * \boldsymbol{T}_{b}=0 ; b=1,2, \ldots, \sigma\right\}, \quad \operatorname{dim} \mathfrak{C}=n-\sigma,
$$

where $R^{n}=\mathscr{C} \oplus \mathfrak{C}^{\perp},\left(\mathscr{C}^{\perp}\right)^{\perp}=\mathscr{G}$. Thus the condition (3.6) and the definition (3.8) can be summarized in the following

THEOREM 2. - The charge of the atomic substances of a reacting mixture is conserved if and only if the reaction vector lies in the $n-\sigma$-dimensional charge subspace of the constituent space; i.e.

$$
\stackrel{+}{\boldsymbol{w}} \in \mathfrak{G} \subset \boldsymbol{R}^{n}
$$

The simultaneous validity of $(3.2)$ and $(3.6)$ for the reaction vector $\stackrel{+}{w}$ imply that $\dot{w} \in \mathcal{S} \cap \mathcal{C}$. The linear space $S \cap \mathcal{C}$ is obviously a linear subspace of $R^{n}$ and it is endowed with the same inner product "**; then it may be called restricted reaction subspace. As far as its dimension $d=\operatorname{dim}(\delta \cap \mathfrak{Z})$ is concerned we can say nothing else than: $1 \leqslant d \leqslant n-\sigma^{(3)}$.

In conclusion we can state.

THEOREM 3. - The mass and the charge of the atomic substances of a reacting mixture are conserved if and only if the reaction vector lies in the d-dimensional restricted reaction subspace of the constituent space, where d may vary from 1 to $n-\sigma$; i.e.

$$
\stackrel{\dot{w}}{\mathrm{w}} \mathrm{S} \cap \mathscr{G} \subset R^{n}
$$

REMARK 1. - It is noteworthy to point out that the axioms of the conservation of mass and of (free) charge for the atomic substance, already synthetically expressed by the 3-rd theorem, can also assume two other different interesting forms. More

$\left(^{3}\right)$ The impossibility of assigning an exact value of the dimension of the restricted reaction space agrees also with a quantum-mechanical description. It is well known that, from this point of view, the presence of an external perturbation (such as an electromagnetic field) on a chemical reaction may only remove degeneracies of the energy levels and may forbid possible transitions, thus reducing the dimension of the reaction vector $w$. In this connection, see any exposition of the quantum mechanical "perturbation theory , described in all the textbooks on Quantum Mechanics. 
precisely, the two eq. (2.21) can be rewritten in the following way

$$
\stackrel{+}{\boldsymbol{w}} * \boldsymbol{M}=0 \quad \text { and } \quad \stackrel{+}{\boldsymbol{w}} * Q=0,
$$

where $\boldsymbol{M}$ and $\boldsymbol{Q}$ are called "mass vector" and "charge vector" respectively. As one can easily verify, $\boldsymbol{M}=\sum_{\mathbf{1}}^{n} M^{a} \boldsymbol{e}_{a}=\sum_{1}^{\sigma} A^{b} \boldsymbol{S}_{b}$ and $\boldsymbol{Q}=\sum_{1}^{n} Q^{a} \boldsymbol{M}^{a} \boldsymbol{e}_{a}=\sum_{1}^{\sigma} A^{b} \boldsymbol{T}_{b} ;$
whence $\boldsymbol{M} \in \mathcal{S}^{\perp}$ and $\boldsymbol{Q} \in \mathcal{C}^{\perp}$.

REMARK 2. When the mixture has no interaction with electromagnetic fields, then the axion of the conservation of (free) charge is always satisfied, in the sense that $Q^{a} \equiv 0, a=1,2, \ldots, n$.

In this case we find $\mathcal{C}^{\perp} \equiv\{0\}$ and $\mathscr{C} \equiv R^{n}$, then the second theorem becomes meaningless whyle the third theorem becomes identical to the first one already proved by R. M. BowEN.

Now the foundamental equation (2.19) takes the form

$$
\dot{\boldsymbol{w}}=\stackrel{+}{\boldsymbol{w}}
$$

which ensures that the time derivative of the concentration vector is in the restricted reaction subspace.

If we generalize a classical idea of De Donder and if we recall the procedure adopted by Bowen in refs. [14], [16], it is easy to show that the so called "extent of reaction vector " $\boldsymbol{W}=\boldsymbol{w}-\boldsymbol{w}_{0}$ is in $S \cap \mathfrak{C}$; obviously $\boldsymbol{w}_{0}$ is the value of $\boldsymbol{w}$ at some reference time and, by eq. $(2.20)$, it must be chosen in such a way that $\boldsymbol{w}_{0} * M=1$ holds.

The condition for the extent of reaction vector $W$ to belong to the restricted reaction subspace can be furtherly improved. In fact the restrictions $(2.20)$ both for $w$ and for $w_{0}$, namely

$$
\boldsymbol{w} * \boldsymbol{M}=1 \quad \text { and } \quad \boldsymbol{w}_{0} * \boldsymbol{M}=1
$$

imply respectively

$$
0<w_{a} \leqslant \frac{1}{\boldsymbol{M}^{a}} \quad \text { and } \quad 0<w_{0 \sigma} \leqslant \frac{1}{\boldsymbol{M}^{a}} \quad a=1,2, \ldots, n
$$

whence

$$
0<w * e_{n} \leqslant \frac{1}{M^{a}} \quad \text { and } \quad 0<w_{0} * e_{o} \leqslant \frac{1}{M^{\alpha}} \quad a=1,2, \ldots, n
$$

Therefore the extent of reaction vector $W$ must belong, a priori, to a bounded closed subset 3 of the constituent space, defined by

$$
\zeta \equiv\left\{\boldsymbol{W} \in R^{n}:\left|W * e_{a}\right| \leqslant \frac{1}{M^{a}} ; \quad a=1,2, \ldots, n\right\}
$$


If we now recall that $S \cap \mathcal{C}$ is a linear subspace of $R^{n}$, we are able to introduce in this intersection a suitable basis $\left(^{4}\right)\left\{\boldsymbol{i}_{1}, \boldsymbol{i}_{2}, \ldots, \boldsymbol{i}_{d} ; d \in[1, n-\sigma]\right\}$ such that

$$
\operatorname{S\cap } \mathfrak{C} \equiv L\left\{i_{p}: p=1,2, \ldots, d ; d \in[1, n-\sigma]\right\}
$$

Taking the previous basis into account, the restrictions (3.12) provide upper limitations for the components of $W$ also in $\boldsymbol{S} \cap \mathcal{G}$. More precisely, we conclude that the extent of reaction vector must lie in a bounded closed subset 3 of the restricted reaction subspace, which is so defined

$$
\mathcal{Z} \equiv\left\{W \in S \cap \mathcal{G}:\left|W * i_{p}\right| \leqslant \frac{1}{\mathcal{M}^{p}} ; p=1,2, \ldots, d ; d \in[1, n-\sigma]\right\}
$$

in (3.17) $1 / \mathcal{M}^{p}=\sum_{1}^{n} \lambda_{p}^{a}\left(1 / M^{a}\right)$ and $\left\|\lambda_{p}^{a}\right\|$ is the transformation matrix between the basis in $R^{n}$ and the basis in $\delta \cap \mathcal{C}$.

The main result of this section can be summarized in the following theorem, which emproves the 3-rd theorem and embodies the fundamental equation (3.11).

THEOREM 4. - The mass and the charge of the atomic substance of a reacting mixture are conserved if and only the extent of reaction vector belongs to a bounded closed subset of the restricted reaction subspace of the constituent space, i.e.:

$$
W \in 3 \subset S \cap \mathscr{C} \subset R^{n}
$$

Moreover the conservation of mass and charge for the whole mixture is ensured if and only if:

$$
\dot{\boldsymbol{W}}=\stackrel{\dot{\boldsymbol{w}}}{.}
$$

At this point, we are able to propose a definition of the term chemical reaction as it applies to our discussion. More exactely we refer to a chemical reaction as to a onedimensional subspace of the restricted reaction space. In this connection we can give the appropriate definition for the indipendence of a collection

$$
\left\{\mathfrak{f}_{i}: i=1,2, \ldots, k \leqslant d ; \operatorname{dim} \mathfrak{T}_{i}=1\right\}
$$

of chemical reactions; namely we say that $k$ chemical reactions are indipendent if

$$
\mathfrak{S}_{i} \cap \sum_{\substack{1 \\ j \neq j}}^{d} \mathfrak{s}_{j}=\{\mathbf{0}\} \quad i=1,2, \ldots, k \leqslant d ; d \in[1, n-\sigma],
$$

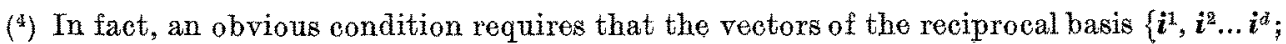
$d \in[1, n-\sigma]\}$ satisfy $i_{p} * \boldsymbol{i}^{\alpha}=\delta_{p}^{q}$. 
where $\{0\}$ is the null chemical reaction. Now a theorem of linear algebra [6], [7] asserts that there exist only $d \leqslant[1, n-\sigma]$ indipendent chemical reactions in a $n$-constituent mixture $\left(^{(5)}\right.$; since $\operatorname{dim}(S \cap \mathcal{C})=d \in[1, n-\sigma]$ we have the following direct sum decomposition

$$
\operatorname{S\cap T}=\bigoplus_{i=1}^{d} \mathfrak{S}_{i} \quad d \in[1, n-\sigma]
$$

These final statements find a deep justification also from a quantum-mechanical point of view, because the reduction of the dimension for the vector $W$ allows a number of indipendent reaction in a "polarized" mixture (i.e. with electromagnetic interactions) which is less or, at most, equal to the number of indipendent reactions in the same mixture without any electromagnetic effect (see the footnote (3) of this section).

Further details and eomments on the rational stoichiometry of "polarized " mixtures (i.e. mixtures subject to electromagnetic fields) will be examined in a future paper.

\section{4. - Field and energy equations for the constituents and for the mixture. Rate equa- tion and processes.}

i) In the first part of this section we consider the basic laws ruling the electromagnetic field in a non diffusing reacting mixture of stationary rigid materials.

If we confine our attention to an arbitrary single constituents $\mathcal{R}_{a} \subset \mathcal{E}(a=1,2, \ldots, n)$ and if we adopt the point of view of P. D. KELLY (see the 4-th sect. of ref. [9]), the two balance laws for the electromagnetic field are assumed to be:

$$
\frac{d}{d t} \int_{s_{a}} \boldsymbol{B}_{a} \cdot \boldsymbol{n}_{a} d s+\int_{\partial s_{a}} \boldsymbol{E}_{a} \cdot d \boldsymbol{x}=\int_{s_{\alpha}} \stackrel{+}{\boldsymbol{b}_{a}} \cdot \boldsymbol{n}_{a} d s \quad a=1,2, \ldots, n
$$

and

$$
\frac{d}{d t} \int_{s_{a}} \boldsymbol{D}_{a} \cdot \boldsymbol{n}_{a} d s-\int_{\delta s_{a}} \boldsymbol{H}_{a} \cdot d \boldsymbol{x}+\int_{\delta_{a}} \boldsymbol{j}_{a} \cdot \boldsymbol{n}_{a} d s=\int_{s_{a}} \dot{\boldsymbol{d}}_{a} \cdot \boldsymbol{n}_{a} d s \quad a=1,2, \ldots, n,
$$

for every "regular" open surface $s_{a} \subset \Re_{a}$ with unit exterior normal vector $\boldsymbol{n}_{a}$.

In eqs. (4.1) and (4.2)-which generalize the classical Faraday's law and Ampere's law respectively, when chemical reactions occur $-\boldsymbol{E}_{a}$ is the electric intensity, $\boldsymbol{H}_{a}$ is the magnetic intensity, $\boldsymbol{D}_{a}$ is the electric induction, $\boldsymbol{B}_{a}$ is the magnetic induction and $\boldsymbol{j}_{a}$ the electric current of the constituent $\mathfrak{R}_{a}$ of the mixture $R$. Moreover, we have introduced the two "electromagnetic supply" vectors $\dot{b}_{a}$ and $\dot{\boldsymbol{d}}_{a}$, which may be

(5) Notice that, when no interaction with electromagnetic fields is considered, eqs. (3.21) and (3.22) reduce to the ones already deduced by $R$. M. Bowen in ref. [16]. 
useful when the fundamental entities of the electromagnetic field are split up into pieces to be associated with each constituent and which take rise from the presence of chemical reactions. Obviously all the fields are vector valued functions of place $\boldsymbol{x}$ and of time $t$, with $(x, t) \in \mathfrak{R}_{a} \times(-\infty, \infty)$.

When suitable smoothness conditions are satisfied, the two integral equations (4.1) and (4.2) become equivalent to the differential equations

$$
\dot{\boldsymbol{B}}_{a}+\operatorname{rot} \boldsymbol{E}_{a}=\stackrel{+}{\boldsymbol{b}}_{a} \quad a=1,2, \ldots, n
$$

and

$$
\dot{\boldsymbol{D}}_{a}-\operatorname{rot} \boldsymbol{H}_{a}+\dot{\boldsymbol{j}}_{a}=\stackrel{+}{\boldsymbol{d}}_{a} \quad a=1,2, \ldots, n,
$$

respectively.

It is well known that the possibility of deducing the correct balance equations of the electromagnetic field for the whole mixture (i.e. the classical Maxwell equations) is strictly related, as it is usual in the modern theory of mixtures, to well posed definitions and to physically meaningful axioms.

We assume the following two axioms of electromagnetic balance for mixtures

$$
\sum_{\mathbf{1}}^{n} \dot{b}_{a}=\mathbf{0} \quad \text { and } \quad \sum_{1}^{n} \stackrel{+}{d}_{a}=\mathbf{0}
$$

with $(x, t) \in \mathcal{R} \times(-\infty, \infty)$. In fact, by (4.4), eqs. (4.2) and (4.3) yield easily

$$
\dot{B}+\operatorname{rot} E=\mathbf{0}
$$

and

$$
\dot{\boldsymbol{D}}-\operatorname{rot} \boldsymbol{H}+\boldsymbol{j}=\mathbf{0},
$$

where the electric intensity $\boldsymbol{E}$, the magnetie intensity $\boldsymbol{H}$, the electric induction $\boldsymbol{D}$ the magnetic induction $B$ and the electric current $\boldsymbol{j}$ are defined by

$$
\boldsymbol{E}=\sum_{1}^{n} \boldsymbol{E}_{a}, \quad \boldsymbol{H}=\sum_{\mathbf{1}}^{n} \boldsymbol{H}_{a}, \quad \boldsymbol{D}=\sum_{1}^{n}{ }_{a} \boldsymbol{D}_{a}, \quad \boldsymbol{B}=\sum_{1}^{n} \boldsymbol{B}_{a}, \quad \boldsymbol{j}=\sum_{1}^{n} \boldsymbol{j}_{a},
$$

with $(x, t) \in \mathfrak{R} \times(-\infty, \infty)\left({ }^{1}\right)$

(1) Further details and developments on this subject can be found in the exhaustive analysis given by P. D. KELLY in ref. [9]. In his paper P. D. KELLY extended the main leading ideas of C. A. TruespenL [1], [2] so as to include, besides electromagnetio interactions, diffusion effects, couple stresses and singular surfaces.

Notice that the presence of an electric current $j$ does not contradict, in general, the assumption on the absence of diffusion, 
ii) At this point we need a balance law for the energy of our mixture consisting of non diffusing reacting stationary rigid materials, where also electromagnetic fields are taken into account.

We denote with $\varepsilon_{a}$ the energy density and with $r_{a}, \boldsymbol{q}_{a}, \boldsymbol{S}_{a}$ the heat supply, the heat flux vector and the electromagnetic flux vector respectively of an arbitrary constituent $\mathfrak{R}_{a} \subset \mathcal{E}(a=1,2, \ldots, n)$. If $\dot{\varepsilon}_{a}$ is the growth of energy due to chemical reactions, the following energy balance law

$$
\frac{d}{d t} \int_{v_{a}} \varrho_{a} \varepsilon_{a} d v-\int_{v_{a}} \varrho_{a} r_{a} d v+\int_{\partial v_{a}}\left(\boldsymbol{q}_{a}+\boldsymbol{S}_{a}\right) \cdot \boldsymbol{n}_{a} d s=\int_{v_{a}} \varrho_{a}^{+} \varepsilon_{a} d v, \quad a=1,2, \ldots, n,
$$

is assumed to hold for every regular subregion $v_{a} \subset \Re_{a}$ with closed regular boundary $\partial v_{a}$. When singular points and surfaces in each constituent are excluded, the integral law (4.8) takes the equivalent local form

$$
\left(\dot{\varrho}_{a} \varepsilon_{a}\right)-\varrho_{a} r_{a}+\operatorname{div}\left(\boldsymbol{q}_{a}+\boldsymbol{S}_{a}\right)=\varrho^{+} \dot{\varepsilon}_{a} \quad a=1,2, \ldots, n
$$

with $(\boldsymbol{x}, t) \in \Re_{a} \times(-\infty, \infty)$.

Now we are able to obtain a correct energy balance equation (i.e. the first law of thermodynamics) for the whole mixture if the axiom of energetic balance for the mixture, namely

$$
\sum_{1}^{n}{ }_{1}^{+}{ }_{\varepsilon}=0
$$

is considered to be true when $(x, t) \in R \times(-\infty, \infty)$.

Thus eqs. (4.9), together with (4.10), yield the well known energy equation for a simple stationary rigid medium

$$
\dot{\varepsilon}-\boldsymbol{r}+\operatorname{div}(\boldsymbol{q}+\boldsymbol{E} \wedge \boldsymbol{H})=\mathbf{0} .
$$

Obviously in (4.11) the following suitable definitions have been introduced

$$
\varepsilon=\sum_{1}^{n} \varrho_{a} \varepsilon_{a}, \quad r=\sum_{1}^{n} \varrho_{a} r_{a}, \quad \boldsymbol{q}=\sum_{1}^{n} \boldsymbol{q}_{a} \quad \text { and } \quad \boldsymbol{S}=\sum_{1}^{n} \boldsymbol{S}_{a} \equiv \boldsymbol{E} \wedge \boldsymbol{H}\left({ }^{2}\right)
$$

with $(\boldsymbol{x}, t) \in \mathfrak{R} \times(-\infty, \infty)$.

$\left({ }^{2}\right)$ Whyle defs. $(4.12)_{1},(4.12)_{\mathrm{a}},(4.12)_{3}$ are familiar, def. (4.12) ${ }_{4}$ deserves some comments. In fact, even if we cannot given an explicit expression for $\boldsymbol{S}_{a}$ (e.g. $\boldsymbol{S}_{a} \neq \boldsymbol{E}_{a} \wedge \boldsymbol{H}_{a}$ !), we are "physically" compelled to assign to $S$ the form $\boldsymbol{E} \wedge \boldsymbol{H}$, where $\boldsymbol{E}$ and $\boldsymbol{H}$ are given by (4.7) and $(4.7)_{2}$ respectively. 
If we adopt a well know procedure we can deduce two formally different expressions for eq. (4.11); more precisely, recalling eqs. (4.5), (4.6), we find easily

$$
\dot{\varepsilon}=r-\operatorname{div} \boldsymbol{q}+\boldsymbol{E} \cdot \dot{\boldsymbol{D}}+\boldsymbol{H} \cdot \dot{\boldsymbol{B}}+\boldsymbol{j} \cdot \boldsymbol{E}
$$

and

$$
\dot{\chi}=r-\operatorname{div} \boldsymbol{q}-\boldsymbol{D} \cdot \dot{\boldsymbol{E}}-\boldsymbol{B} \cdot \dot{\boldsymbol{H}}+\boldsymbol{j} \cdot \boldsymbol{E}
$$

where $\chi=\varepsilon-\boldsymbol{E} \cdot \boldsymbol{D}-\boldsymbol{H} \cdot \boldsymbol{B}$ is the Helmotz enthalpy density of the mixture.

For the sake of convenience, the two energy equations $(4.13)_{a}$ and $(4.13)_{b}$ can be rewritten in a slightly different form if we introduce

$$
\psi=\varepsilon-\theta \eta \quad \xi=\chi-\theta \eta
$$

as the free energy density and the Gibbs free enthalpy density of the mixture, where $\eta$ denotes the entropy density and $\theta$ is the common temperature of each consistituent of the mixture (3). In fact, by defs. (4.14), eqs. $(4.13)_{a}$ and $(4.13)_{b}$ become

$$
(\psi \dot{+} \theta \eta)=r-\operatorname{div} \boldsymbol{q}+\boldsymbol{E} \cdot \dot{\boldsymbol{D}}+\boldsymbol{H} \cdot \dot{\boldsymbol{B}}+\boldsymbol{j} \cdot \boldsymbol{E}
$$

and

$$
(\xi \dot{\dagger} \theta \eta)=r-\operatorname{div} \boldsymbol{q}-\boldsymbol{D} \cdot \dot{\boldsymbol{E}}-\boldsymbol{B} \cdot \dot{\boldsymbol{H}}+\boldsymbol{j} \cdot \boldsymbol{E}
$$

respectively $\left({ }^{4}\right)$.

Now we are ready to propose the fundamental definitions of "process" and of "admissible process " in a non diffusing reacting mixtures of stationary rigid materials with electromagnetic fields.

Definition 1. - A collection of twelve fields $\boldsymbol{E}, \boldsymbol{H}, \boldsymbol{B}, \boldsymbol{D}, \boldsymbol{q}, \boldsymbol{j}, r, \xi, \eta, \theta, \stackrel{+}{\boldsymbol{w}}, \boldsymbol{W}$ -each one a scalar or vector valued function on $R \times(-\infty, \infty)$-is called a process on $R \times(-\infty, \infty)$ if and only if is compatible with the field equations (4.5) and (4.6), with the energy equation in the form $(4.15)_{b}$ and with the chemical equation (3.20).

Defrnition 2. - A collection of twelve fields $\boldsymbol{E}, \boldsymbol{H}, \boldsymbol{B}, \boldsymbol{D}, \boldsymbol{q}, \boldsymbol{j}, r, \xi, \eta, \theta, \stackrel{+}{\boldsymbol{w}}, \boldsymbol{W}$, -each one defined on $R \times(-\infty, \infty)$-is said an admissible process on $R \times(-\infty, \infty)$ if and only if it is a process on $R \times(-\infty, \infty)$ and it is also consistent with the so called "constitutive relations " of the mixture.

$\left({ }^{3}\right)$ Notice that $\eta=\sum_{1}^{n} \varrho_{a} \eta_{a}$ and $\psi=\sum_{1}^{n} \varrho_{a} \psi_{a}$, with $\psi_{a}=\varepsilon_{a}-\theta \eta_{a}$; whyle an analogous factorization for $\chi$ and $\xi$ is not allowed because of the presence of electromagnetio terms.

(4) According to the spirit of the modern theory of mixtures, our main field and energy equations (4.5), (4.6) and (4.11) are quite analogous to the ones proposed by B. D. CoLEMAN and $\mathbf{E}$. H. DrLL in their thermodynamic theory of electromagnetic fields in stationary rigid materials [24, Sect. 1], [25, Sect. 1]. 
In our paper, we assume constitutive relations of the following type:

$$
\begin{array}{ll}
\xi=\hat{\xi}(\theta, \boldsymbol{g}, \boldsymbol{E}, \boldsymbol{H}, \boldsymbol{W}), & \eta=\hat{\eta}(\theta, \boldsymbol{g}, \boldsymbol{E}, \boldsymbol{H}, \boldsymbol{W}), \\
\boldsymbol{D}=\hat{\boldsymbol{D}}(\theta, \boldsymbol{g}, \boldsymbol{E}, \boldsymbol{H}, \boldsymbol{W}), & \boldsymbol{B}=\hat{\boldsymbol{B}}(\theta, \boldsymbol{g}, \boldsymbol{E}, \boldsymbol{H}, \boldsymbol{W}), \\
\boldsymbol{q}=\hat{\boldsymbol{q}}(\theta, \boldsymbol{g}, \boldsymbol{E}, \boldsymbol{H}, \boldsymbol{W}), & \boldsymbol{j}=\hat{\boldsymbol{j}}(\theta, \boldsymbol{g}, \boldsymbol{E}, \boldsymbol{H}, \boldsymbol{W}),
\end{array}
$$

and

$$
\stackrel{+}{\boldsymbol{w}}=\hat{\boldsymbol{f}}(\theta, \boldsymbol{g}, \boldsymbol{E}, \boldsymbol{H}, \boldsymbol{W})
$$

where $g=\operatorname{grad} \theta$ is the temperature gradient ( $\left.{ }^{5}\right)$.

If we denote with $R$ the set of all reals and with $\mathcal{V}_{3}$ the space of all ordinary threedimensional vectors, the functions $\hat{\xi}, \hat{\eta}, \hat{\boldsymbol{D}}, \hat{\boldsymbol{B}}, \hat{\boldsymbol{q}}, \hat{\boldsymbol{j}}, \hat{\boldsymbol{f}}$ are defined and continuous with all their derivatives in a given open set $\mathcal{A}$ of $(R-\{0\}) \times \mathcal{V}_{3} \times \mathcal{V}_{3} \times \mathcal{V}_{3} \times 3$, with values in $R, R, V_{3}, V_{3}, V_{3}, V_{3}$ and $\delta \cap \mathcal{G}$ respectively $\left(^{6}\right)$.

The last of the constitutive relations (4.16), together with the chemical equation $(3.20)$, yields to the "rate equation " for the extent of reaction vector; i.e.

$$
\dot{W}=\hat{f}(\theta, g, \boldsymbol{E}, \boldsymbol{H}, \boldsymbol{W})
$$

Equation (4.18) is an ordinary differential equation for $W$ when the thermal field $\theta=\theta(\boldsymbol{x}, t)$, the electric intensity field $\boldsymbol{E}=\boldsymbol{E}(\boldsymbol{x}, t)$ and the magnetic intensity field $\boldsymbol{H}=\boldsymbol{H}(\boldsymbol{x}, t)$ are given in $R \times(-\infty, \infty)$; in addition an initial value for $\boldsymbol{W}$ must be assigned ( 7 ).

As for as the "response function " $\hat{f}$ in the r.h.s. of eq. (4.17) is concerned, it is customary to make two assumptions whose importance will become clear in the following two sections.

Assumption 1. - The function $\hat{f}: \mathcal{A} \rightarrow S \cap \mathfrak{G}$ is such that it vanishes for at least an element of the form $(\theta, \mathbf{0}, \mathbf{0}, \boldsymbol{H}, \boldsymbol{W}) \in \mathcal{A}$.

Assuription 2. - The funetion $\hat{f}: \mathfrak{A} \rightarrow S \cap \mathcal{C}$ is such that the rate equation (4.17), associated with the initial condition $W\left(x, t_{0}\right)=W_{0} \in 3$ and with sufficiently smooth

(5) When no chemical reaction occurs, we have $\boldsymbol{W}=\mathbf{0}$. Thus the constitutive relations reduce to the ones proposed by B. D. Coleman and E. H. Dms in the 4.th Sect. of ref. [24].

${ }^{(8)}$ The usual restrietions $\theta(\boldsymbol{x}, t)>0$ is dropped as there are systems, interacting with electromagnetic fields, which exhibit negative temperatures; see refs. quoted in [224], [25], [26].

(7) It is worth while to remark that equations of the type (4.17) are familiar in many theories of materials with "internal variables ", where the meaning of the quantities involved is different according to the various cases under consideration (e.g. Eriksen's theory of anisotropic fluids, Kratochvil's theory of elasto-plastic behavior of certain materials ete.).

An exaustive and general exposition of the internal variables forma.ism can bo found in the excellent paper by B. D. Coleman and M. E. GurTin [11]. 
fields, has a unique solution on some interval $\left[t_{0}, t_{0}+\Delta ; \Delta>0\right]$, for every $x \in \Re$ and for every arbitrary $t_{0} \in(-\infty, \infty)$.

When we consider the other "response functions " in (4.16) it is convenient to lay down a further assumption which will be af use in the sequel.

Assumption 3. - The "response functions" $\hat{\xi}, \hat{\eta}: A t \rightarrow R$ and $\hat{\boldsymbol{D}}, \hat{\boldsymbol{B}}, \hat{\boldsymbol{q}}, \hat{\boldsymbol{j}}: \mathfrak{A} \rightarrow \cup_{3}$ must satisfy the so called "solvability condition" in order to ensure a strict validity of the procedure which will be adopted in the next section (see $[24, \S 3],[25, \S 4]$ for a more general situation).

In our case the above assumption is generally satisfied as a consequence of the regularity of the response funetions and of the arbitrariness of the heat supply field $r(x, i)$. In fact, when we consider a small subregion $R_{x} \subset \mathcal{R}$ containing a fixed material point $x$ of the mixture, eqs. (4.5), (4.6), (4.15) $)_{3}$ together with (4.16) and (4.17) -with $W=W_{0}$ throughout-give rise to a sistem of seven second-order partial differential equations with analytic coefficients. Now the Cauchy-Kowalewski theorem provides the existence and the uniqueness of the solution when the set of initial data is suitably assigned. More precisely, if we give $\theta\left(\boldsymbol{x}, t_{0}\right)=\theta_{0}, \boldsymbol{E}\left(\boldsymbol{x}, t_{0}\right)=\boldsymbol{E}_{0}$, $\boldsymbol{H}\left(\boldsymbol{x}, t_{0}\right)=\boldsymbol{H}_{0}$ and if we set $\dot{\theta}\left(\boldsymbol{x}, t_{0}\right)=c, \dot{\boldsymbol{E}}\left(\boldsymbol{x}, t_{0}\right)=\boldsymbol{M}, \dot{\boldsymbol{H}}\left(\boldsymbol{x}, t_{0}\right)=\boldsymbol{N}$-with $\theta_{0}$ an arbitrary constant positive scalar, $c$ an arbitrary constant scalar and $\boldsymbol{E}_{0}, \boldsymbol{H}_{\mathbf{0}}, \boldsymbol{M}, \boldsymbol{N}$ arbitrary constant vectors-we can write:

$$
\begin{array}{llll}
\theta=\theta\left(\boldsymbol{x}, t \mid \theta_{0}, \boldsymbol{E}_{0}, \boldsymbol{H}_{0}, c, \boldsymbol{M}, \boldsymbol{N}\right) & t \in\left[t_{0}, t_{0}+\delta\right] & 0<\delta \leqslant \Delta, \\
\boldsymbol{E}=\boldsymbol{E}\left(\boldsymbol{x}, t \mid \theta_{0}, \boldsymbol{E}_{0}, \boldsymbol{H}_{0}, c, \boldsymbol{M}, \boldsymbol{N}\right) & t \in\left[t_{0}, t_{0}+\delta\right] & 0<\delta \leqslant \Delta, \\
\boldsymbol{H}=\boldsymbol{H}\left(\boldsymbol{x}, t \mid \theta_{0}, \boldsymbol{E}_{0}, \boldsymbol{H}_{0}, c, \boldsymbol{M}, \boldsymbol{N}\right) & t \in\left[t_{0}, t_{0}+\delta\right] & 0<\delta \leqslant \Delta,
\end{array}
$$

It appears reasonable, when $\operatorname{det}\left\|\partial(\theta, \boldsymbol{E}, \boldsymbol{H}) / \partial\left(\theta_{0}, \boldsymbol{E}_{0}, \boldsymbol{H}_{0}\right)\right\| \neq 0$, to assert that one can indipendently choose $(\theta, \boldsymbol{H}, \boldsymbol{E}), \dot{\boldsymbol{g}}$ and $\boldsymbol{g}$ at a given fixed material point $\boldsymbol{x}$ of the mixture in the sense that we can write

$$
\boldsymbol{g}\left(\boldsymbol{x}, t_{0}\right)=\boldsymbol{g}_{0} \quad \text { and } \quad \dot{\boldsymbol{g}}\left(\boldsymbol{x}, t_{0}\right)=\boldsymbol{k}
$$

with $\boldsymbol{g}_{0}, \boldsymbol{k}$ constant and arbitrary vectors.

Obviously the remark outlined in this 3-rd assumption must taken into account also for refs. [26] and [30].

\section{5. - Entropy inequality and its consequences. Electromagnetic chemical afflnity.}

One of the most discussed problems in the modern theory of mixtures is the construction of a meaningful expression for the growth of entropy in each constituent and the consequent assumption of a suitable "dissipation" entropy inequality for the mixture as a whole. Whyle the axioms introduced in the previous sections (namely $(2.16),(2.18),(4.4)_{1,2}$ and $(4.10)$ ) are generally accepted, some difficulties 
arise when the second law of thermodynamics is to be applied to mixtures. Anyhow, in our paper, the main source of theoretical troubling dissappears since we confine our attention to non diffusing mixtures and we examine electromagnetic interactions only with stationary rigid materials ( ${ }^{1}$ ).

Thus, if we denote with $\dot{\eta}_{a}$ the entropy supply of the arbitrary component $\Re_{a} \subset \&(a=1,2, \ldots, n)$ due to chemical reactions, let us assume the following law of balance for entropy

$$
\frac{d}{d t} \int_{v_{a}} \varrho_{a} \eta_{a} d v-\int_{v_{a}} \varrho_{a} \frac{r_{a}}{\theta} d v+\int_{\partial v_{a}} \frac{\boldsymbol{q}_{a}}{\theta} \cdot \boldsymbol{n}_{a} d s=\int_{v_{a}} \varrho_{\eta_{a}}^{+} d v \quad a=1,2, \ldots, n
$$

for every regular subregion $v_{a} \subset \mathcal{R}_{a}$ with closed regular boundary $\partial v_{a}\left({ }^{2}\right)$. The local form of eq. (5.1) is given by

$$
\left(\varrho_{a} \eta_{a}\right)-\varrho_{a} \frac{r_{a}}{\theta}+\operatorname{div}\left(\frac{\boldsymbol{q}_{a}}{\theta}\right)=\varrho_{\eta_{a}}^{+} \quad a=1,2, \ldots, n
$$

with $(x, t) \in \Re_{a} \times(-\infty, \infty)$.

Following a well posed conjecture of $C$. A. Truesdeut and $R$. M. Bowen, we can postulate the axiom of entropic dissipation for the mixture, i.e.

$$
\sum_{1}^{n}{ }_{1}^{+} \dot{\eta}_{a} \geqslant 0
$$

where $(x, t) \in R \times(-\infty, \infty)$.

By the defs. (4.12) $)_{2,3}$ of $r$ and $\boldsymbol{q}$ respectively and by the definition of $\eta$ given in the 3-rd footnote of the previous section, (5.2) and (5.3) yield easily

$$
\dot{\eta}-\frac{r}{\theta}+\operatorname{div}\left(\frac{q}{\theta}\right) \geqslant 0
$$

The above entropy inequality, which has a form analogous to the one proposed by B. D. Coleman and E. H. DILL in refs. [24], [25], is to be regarded as the differential expression of the second law of thermodynamics for the mixture in exam. Before going further, the entropy inequality can be usefull rearranged by eliminating the mixture heating $r$ with the aid of one of the energy equations. In fact (5.4), together

(1) An interesting comparison of the various theories, up to date till 1969 , is given in the 5 -th and in the 6-th lectures of Truesdell's book [18]; more recent contributions can be found in the references quoted in [22], [23].

$\left({ }^{2}\right)$ The absence of a flux of $\boldsymbol{S}_{a} / \theta(a=1,2, \ldots, n)$ in $(5.1)$ could surprise from a merely mathematical point of view, but it is in complete agreement with the physical meaning of the quantities involved. In fact, the first and the second integral in the r.h.s. eq. (5.1) represent contributions to the so-called irreversible sheat (naturally related to the entroy concept), whyle the flux of $\boldsymbol{S}_{a} / \theta$ does not give any contribution of this type. 
with $(4.15)_{b}$, becomes the reduced entropy inequality:

$$
\theta(\dot{\xi}+\eta \dot{\theta})+\theta(\boldsymbol{D} \cdot \dot{\boldsymbol{E}}+\boldsymbol{B} \cdot \dot{\boldsymbol{H}}-\boldsymbol{j} \cdot \boldsymbol{E})+\boldsymbol{q} \cdot \boldsymbol{g} \leqslant 0
$$

As it is usual in Continuum Thermomechanics, we adopt now the Colemen-Noll interpretation of the second law [8], [18] by laying down the following assumption:

The entropy inequality (5.4), or the reduced entropy inequality (5.5), must hold for every admissible process in the mixture in each point $\boldsymbol{x} \in \mathcal{R}$ and at any instant $t \in(-\infty, \infty)$.

If we recall the constitutive relations $(4.16)$ and if we notice that

$$
\begin{aligned}
\dot{\xi} & =\frac{d}{d t} \hat{\xi}(\theta, \boldsymbol{g}, \boldsymbol{E}, \boldsymbol{H}, \boldsymbol{W})= \\
& =\partial_{\theta} \hat{\xi} \dot{\theta}+\partial_{\boldsymbol{g}} \hat{\xi} \cdot \dot{\boldsymbol{g}}+\partial_{\boldsymbol{E}} \hat{\xi} \cdot \dot{\boldsymbol{E}}+\partial_{\boldsymbol{H}} \hat{\xi} \cdot \dot{\boldsymbol{H}}+\nabla_{\boldsymbol{W}} \hat{\xi} * \dot{\boldsymbol{W}}\left({ }^{(3)}\right)
\end{aligned}
$$

the reduced inequality (5.5) reads

$$
\begin{aligned}
\theta\left[\left(\partial_{\theta} \hat{\xi}+\hat{\eta}\right) \dot{\theta}+\partial_{\boldsymbol{g}} \hat{\xi} \cdot \dot{\boldsymbol{g}}+\left(\partial_{\boldsymbol{E}} \hat{\xi}+\hat{\boldsymbol{D}}\right) \cdot \dot{\boldsymbol{E}}+\left(\partial_{\boldsymbol{H}} \hat{\xi}+\hat{\boldsymbol{B}}\right) \cdot \dot{\boldsymbol{H}}\right]+ \\
+\hat{\boldsymbol{q}} \cdot \boldsymbol{g}-\theta \boldsymbol{j} \cdot \boldsymbol{E}+\theta \hat{\mathrm{\alpha}_{\mathrm{em}}} * \hat{\boldsymbol{f}} \leqslant 0 .
\end{aligned}
$$

In deriving the inequality (5.6) we have also used the rate equation (4.17) and we have introduced the "electromagnetic chemical affinity" vector

$$
\alpha_{\mathrm{em}}=\hat{\alpha}_{\mathrm{em}}(\theta, \boldsymbol{g}, \boldsymbol{E}, \boldsymbol{H}, \boldsymbol{W})=\nabla_{\boldsymbol{W}} \hat{\xi}(\theta, \boldsymbol{g}, \boldsymbol{E}, \boldsymbol{H}, \boldsymbol{W})
$$

which lies obviously in the restricted reaction space.

Since the inequality (5.6) must hold for every admissible process, we are in a position to verify whether or not it is compatible with the a priori assigned constitutive relations (4.16). At this purpose and for the sake of simplicity, we consider the admissible process in an arbitrary fixed point $x$ of the mixture such that (3-rd as-

(3) Hereafter we denote with $\partial_{g}, \partial_{E}, \partial_{H}$ the usual gradient operator in $\mathcal{U}_{3}$, while $\nabla_{W}$ stands for the analogous operator in the restricted reaction space $\mathcal{S} \cap \mathcal{G}$; more precisely, if $\Psi$ is a scalar or a vector valued function defined on $\mathcal{Z} \subset \mathcal{S} \cap \mathcal{C}$, we can write

for every $N \in \mathfrak{Z}$.

$$
\nabla_{\boldsymbol{W}} \Psi(\boldsymbol{W})[\boldsymbol{U}]=\left[\frac{d}{d s} \Psi(\boldsymbol{W}+s \boldsymbol{U})\right]_{s=0}
$$

When $X$ and $Y$ are two finite dimensional linear spaces, $\mathcal{L}(X, Y)$ is the linear space of all the linear transformations from $X$ to $T$. Therefore we have either $\nabla_{W} \Psi \in \mathcal{L}(R, \mathcal{S} \cap \mathcal{Z})$ or $\nabla_{W} \Psi \in \mathcal{C}\left(\mathcal{U}_{3}, S \cap \mathcal{G}\right)$ according to $\Psi$ is either a scalar or a vector valued function. Since it will be of use in the sequel, we denote with $\mathcal{L}(X)$ the algebra of all the endomorphysms from $X$ to $X$, and with $\mathfrak{L}^{*}(X)$ the group of all the automorphysms from $X$ to $X$, see [6], [7]. 
sumption of the 4 -th section):

$$
\begin{array}{llll}
\theta(\tau)=\theta_{0}+\left(\tau-t_{0}\right) \boldsymbol{c} \neq 0 & \tau \in\left[t_{0}, t_{0}+\delta\right], & 0 \leqslant \delta \leqslant \Delta, \\
\boldsymbol{g}(\tau)=\boldsymbol{g}_{0}+\left(\tau-t_{0}\right) \boldsymbol{k} & \tau \in\left[t_{0}, t_{0}+\delta\right], & 0 \leqslant \delta \leqslant \Delta, \\
\boldsymbol{E}(\tau)=\boldsymbol{E}_{0}+\left(\tau-t_{0}\right) \boldsymbol{M} & \tau \in\left[t_{0}, t_{0}+\delta\right], & 0 \leqslant \delta \leqslant \Delta, \\
\boldsymbol{H}(\tau)=\boldsymbol{H}_{0}+\left(\tau-t_{0}\right) \boldsymbol{N} & \tau \in\left[t_{0}, t_{0}+\delta\right], & 0<\delta \leqslant \Delta,
\end{array}
$$

where $\left(\theta_{0}, \boldsymbol{g}_{0}, \boldsymbol{E}_{0}, \boldsymbol{H}_{\mathbf{v}}\right)$ are a fixed set of arguments in the domain of (4.16) and $(c, k, M, N)$ is any element of $R \times \mathcal{V}_{3} \times \mathcal{V}_{3} \times V_{3}$.

For the process (5.8), (5.6) implies that

$$
\begin{aligned}
\theta(\tau)\left[\left(\partial_{\theta} \hat{\xi}-\hat{\eta}\right) c+\partial_{\boldsymbol{g}} \hat{\xi} \cdot \boldsymbol{k}+\left(\partial_{\boldsymbol{E}} \hat{\xi}+\hat{\boldsymbol{D}}\right) \cdot \boldsymbol{M}+\left(\partial_{\boldsymbol{H}} \hat{\xi}+\hat{\boldsymbol{B}}\right) \cdot \boldsymbol{N}\right]+ \\
+\hat{\boldsymbol{q}} \cdot \boldsymbol{g}(\tau)-\theta(\tau) \hat{\boldsymbol{j}} \cdot \boldsymbol{E}(\tau)+\theta(\tau) \hat{\boldsymbol{\alpha}}_{\mathrm{em}} * \hat{\boldsymbol{f}} \leqslant 0
\end{aligned}
$$

must hold for each $c \in R$ and each $k, M, N \in \mathcal{V}_{3}$.

The smoothness conditions on the constitutive relations allow us to write

$$
\lim _{\tau \rightarrow t_{0}} \psi[\theta(\tau), \boldsymbol{g}(\tau), \boldsymbol{E}(\tau), \boldsymbol{H}(\tau), \boldsymbol{W}(\tau)]=\psi\left(\theta_{0}, \boldsymbol{g}_{0}, \boldsymbol{E}_{0}, \boldsymbol{H}_{0}, W_{0}\right)=\psi_{0}
$$

where $\psi$ is any scalar or vector valued function in the r.h.s. of (5.9) and $W_{0} \in \mathcal{Z}$ is the initial value of $W$, chosen in such a way that the rate equation (4.17) $\mathrm{h} d \mathrm{~s}$ a unique solution for the assigned process (5.8) (see the 2-nd assumption at the and of the 4-th section).

Thus, taking the limit $\tau \rightarrow t_{0}$ in the r.h.s. of (5.9), we find

$$
\begin{aligned}
\theta_{0}\left\{\left[\left(\partial_{\theta} \hat{\xi}\right)_{0}+\hat{\eta}_{0}\right] c+\left(\partial_{\boldsymbol{g}} \hat{\xi}\right)_{0} \cdot \boldsymbol{k}+\left[\left(\partial_{\boldsymbol{E}} \hat{\xi}\right)_{0}+\hat{\boldsymbol{D}}_{0}\right] \cdot \boldsymbol{M}+\left[\left(\partial_{\boldsymbol{H}} \hat{\xi}\right)_{0}+\hat{\boldsymbol{B}}_{0}\right] \cdot \boldsymbol{N}\right\}+ \\
+\hat{\boldsymbol{q}}_{0} \cdot \boldsymbol{g}_{0}-\theta_{0} \hat{\boldsymbol{j}}_{0} \cdot \boldsymbol{E}_{0}+\theta_{0} \hat{\alpha}_{\mathrm{em} 0} * \hat{\boldsymbol{f}}_{0} \leqslant 0
\end{aligned}
$$

for any $c \in R$ and any $\boldsymbol{k}, \boldsymbol{M}, \boldsymbol{N} \in \mathcal{V}_{3}$.

From the arbitrariness and indipendence of $c, k, \boldsymbol{M}, \boldsymbol{N}$, the last inequality yields

$$
\begin{array}{ll}
\hat{\eta}+\partial_{\theta} \hat{\xi}=0, & \partial_{\boldsymbol{g}} \hat{\xi}=0, \\
\hat{\boldsymbol{D}}+\partial_{\boldsymbol{E}} \hat{\xi}=0, & \hat{\boldsymbol{B}}+\partial_{\boldsymbol{H}} \hat{\xi}=0
\end{array}
$$

and

$$
\hat{\boldsymbol{q}} \cdot \boldsymbol{g}-\theta \hat{\boldsymbol{j}} \cdot \boldsymbol{E}+\theta \hat{\alpha}_{\mathrm{em}} * \hat{\boldsymbol{f}} \leqslant 0,
$$

where the subscript " 0 " has been dropped.

These results can be summarized by stating the following main theorem. 
THEOREM 5. - When a mixture of non diffusing reacting stationary rigid materials with electromagnetic fields is described by the constitutive relations (4.16), the entropy inequality holds for all smooth admissible processes if and only if the following conditions are satisfied:

with

$$
\begin{array}{ll}
\xi=\hat{\xi}(\theta, \boldsymbol{E}, \boldsymbol{H}, \boldsymbol{W}), & \eta=\hat{\eta}(\theta, \boldsymbol{E}, \boldsymbol{H}, \boldsymbol{W}) \\
\boldsymbol{D}=\hat{\boldsymbol{D}}(\theta, \boldsymbol{E}, \boldsymbol{H}, \boldsymbol{W}), & \boldsymbol{B}=\hat{\boldsymbol{B}}(\theta, \boldsymbol{E}, \boldsymbol{H}, \boldsymbol{W})
\end{array}
$$

$$
\hat{\eta}=-\partial_{\theta} \hat{\xi}, \quad \hat{\boldsymbol{D}}=-\partial_{\boldsymbol{E}} \hat{\xi}, \quad \hat{\boldsymbol{B}}=-\partial_{\boldsymbol{H}} \hat{\xi} .
$$

Moreover $\hat{\boldsymbol{q}}, \hat{\boldsymbol{j}}, \hat{\boldsymbol{\alpha}}_{\mathrm{em}}$ and $\hat{\boldsymbol{f}}$ must satisfy the «chemical dissipation inequality for thermal and electric conduction»:

$$
\hat{\boldsymbol{q}} \cdot \boldsymbol{g}-\theta \hat{\boldsymbol{j}} \cdot \boldsymbol{E}+\theta \hat{\boldsymbol{\alpha}_{\mathrm{em}}} * \hat{\boldsymbol{f}} \leqslant 0
$$

REMARK 1. - Obvious consequences of (5.12) are

$$
\begin{gathered}
\partial_{\theta} \hat{\boldsymbol{D}}=\partial_{\boldsymbol{E}} \hat{\eta}, \quad \partial_{\theta} \hat{\boldsymbol{B}}=\partial_{\boldsymbol{H}} \hat{\eta}, \quad \partial_{\boldsymbol{H}} \hat{\boldsymbol{D}}=\partial_{\boldsymbol{E}} \hat{\boldsymbol{B}} \\
\partial_{\boldsymbol{E}} \hat{\boldsymbol{D}}=\left(\partial_{\boldsymbol{E}} \hat{\boldsymbol{D}}\right)^{T}, \quad \partial_{\boldsymbol{H}} \hat{\boldsymbol{B}}=\left(\partial_{\boldsymbol{H}} \hat{\boldsymbol{B}}\right)^{T},
\end{gathered}
$$

where $\partial_{\boldsymbol{E}} \hat{\boldsymbol{D}}, \partial_{\boldsymbol{H}} \hat{\boldsymbol{D}}, \partial_{\boldsymbol{E}} \hat{\boldsymbol{B}}, \partial_{\boldsymbol{H}} \hat{\boldsymbol{B}} \in \mathcal{L}\left(\mathcal{V}_{3}, \bigcup_{3}\right)$ and the superscript $" T$ » denote the transpose of linear transformations $\left({ }^{4}\right)$.

REMarK 2. - A classical argument, introduced by B. D. Coleman and W. Noll in ref. [8] and used again by B. D. CoLEMAN and E. H. DILL in refs. [24], [25], cannot be applied to our "chemical dissipation inequality for thermal and electric conduction ".

In fact, from (5.13) we cannot infer that, whenever both the temperature gradient and the electric intensity vanish, neither heat nor current flows. In other words, a correct definition of an "equilibrium state" for the mixture in exam is needed. This question will be discussed in detail in the next section.

REMARK 3. - If we notice that $\hat{\xi}=\hat{\psi}-\boldsymbol{E} \cdot \hat{\boldsymbol{D}}-\boldsymbol{H} \cdot \hat{\boldsymbol{B}}$ is an immediate consequence of the definitions (4.14) and of the constitutive relations $(4.16)_{1,2,3,4}$, the electromagnetic chemical affinity becomes

$$
\hat{\alpha}_{\mathrm{em}}=\nabla_{W} \hat{\psi}-\nabla_{W}(\boldsymbol{E} \cdot \hat{\boldsymbol{D}}+\boldsymbol{H} \cdot \hat{\boldsymbol{B}})=\hat{\alpha}-\left[\left(\nabla_{W} \hat{\boldsymbol{D}}\right) \boldsymbol{E}+\left(\nabla_{W} \hat{\boldsymbol{B}}\right) \boldsymbol{H}\right],
$$

$\left({ }^{4}\right)$ When no chemical reactions occurs (i.e. $W \equiv \mathbf{0}$ ) our theorem 5 gives the same results proved for stationary rigid materials either with "instantaneous " electromagnetic response [24, sect. 4] or with a "quasi " electromagnetic response, provided only steady states are considered [26, sect. 3]. The implications of the "dissipation inequality for thermal and electric conduction" (in the case of absence of chemical effects) has been recently examined for isotropic solids by R. Borghesan I and A. Morro [27], [28]. 
where $\hat{\alpha}=\nabla_{W} \hat{\psi}$ is the "chemical affinity" according to the definition given by R. M. BowEN [14]. In (5.16) the two linear transformations $\nabla_{W} \hat{\boldsymbol{D}}, \nabla_{W} \hat{\boldsymbol{B}}$ belong to $\mathcal{C}\left(\mathcal{V}_{3}, \mathrm{~S} \cap \mathcal{G}\right)$ and then can be represented by two $(d \times 3)$ matrices when the vectors $\boldsymbol{E}$ and $\boldsymbol{H}$ are regarded as column matrices; namely $\nabla_{W} \hat{\boldsymbol{D}} \equiv \| \nabla_{W_{k}} \hat{\boldsymbol{D}}_{i}: k=1,2, \ldots, d$; $i=1,2,3 \|$ and $\nabla_{\boldsymbol{W}} \hat{\boldsymbol{B}} \equiv\left\|\nabla_{W_{k}} \hat{\boldsymbol{B}}_{i}: k=1,2, \ldots, d ; i=1,2,3\right\|$ with $d \in[1, n-\sigma]\left(^{5}\right)$.

When only "weak fields " are involved the constitutive relations (5.11) $)_{2}$ and $(5.12)_{3}$ are assumed to be linear with respect to $\boldsymbol{E}$ and $\boldsymbol{H}$ respectively; i.e.

$$
\hat{\boldsymbol{D}}=\hat{\boldsymbol{x}} \boldsymbol{E}, \quad \hat{\boldsymbol{B}}=\hat{\mu} \boldsymbol{H},
$$

where the dielectric tensor $\hat{x}$ and the magnetic permeability tensor $\hat{\mu}$ depend only on $\theta$ and $W$ and must be symmetric as a consequence of $(5.15)_{1,2}$. In this case, after a simple calculation, the electromagnetic chemical affinity reads

$$
\hat{\boldsymbol{\alpha}}_{\mathrm{em}}=\hat{\boldsymbol{\alpha}}-\left[\left(\nabla_{\boldsymbol{W}} \hat{\boldsymbol{x}}\right) \boldsymbol{E} \otimes \boldsymbol{E}+\left(\nabla_{w} \hat{\boldsymbol{\mu}}\right) \boldsymbol{H} \otimes \boldsymbol{H}\right]
$$

with $\nabla_{W} \hat{x}$, and $\nabla_{W} \hat{\mu} \in \mathcal{L}\left(V_{3} \oplus \mathcal{V}_{3}, S \cap \mathfrak{C}\right)$.

Finally, if $\hat{\boldsymbol{x}}=\hat{\boldsymbol{x} I}$ and $\hat{\boldsymbol{\mu}}=\hat{\boldsymbol{\mu} I}$ (I being the unit tensor) eq. (5.18) reduces to

$$
\begin{aligned}
\hat{\alpha}_{\mathrm{em}} & =\hat{\alpha}-\left[\left(\nabla_{W} \hat{\chi}\right) E^{2}+\left(\nabla_{W} \hat{\mu}\right) H^{2}\right] \cong \\
& \cong \hat{\alpha}_{0}-\left[\left(\nabla_{W} \hat{\chi}\right) E^{2}+\left(\nabla_{W} \hat{\mu}\right) H^{2}\right],
\end{aligned}
$$

which agrees with a classical result [5; cap. XIV].

\section{6. - Equilibrium and thermal equilibrium states. Classical equilibrium.}

As we pointed out in the 2-nd remark at the end of the previous section, the "chemical dissipation inequality for thermal and electric conduction" requires suitable definitions for what we can call an "equilibrium state " of our mixture (1).

First of all, if we set

$$
\begin{aligned}
\Phi(\theta, \mathbf{g}, \boldsymbol{E}, \boldsymbol{H}, \boldsymbol{W})=\hat{\boldsymbol{q}}(\theta, \boldsymbol{g}, \boldsymbol{E}, \boldsymbol{H}, \boldsymbol{W}) \cdot \boldsymbol{g} & -\theta \hat{\boldsymbol{j}}(\theta, \boldsymbol{g}, \boldsymbol{E}, \boldsymbol{H}, \boldsymbol{W}) \cdot \boldsymbol{E}+ \\
& +\theta \hat{\alpha}_{\mathrm{em}}(\theta, \boldsymbol{E}, \boldsymbol{H}, \boldsymbol{W}) * \hat{\boldsymbol{f}}(\theta, \boldsymbol{g}, \boldsymbol{E}, \boldsymbol{H}, \boldsymbol{W}),
\end{aligned}
$$

${ }^{(5)}$ In the absence of electromagnetic fields we have $\hat{\alpha}_{\mathrm{Bm}} \equiv \hat{\alpha}_{0}=\left(\nabla_{W} \hat{\Psi}\right)_{\boldsymbol{E}, \boldsymbol{H}=\boldsymbol{0}}$.

(1) The need for a critical revision of the definitions quoted in standard textbooks-see e.g. [5]-has been stressed by R. M. Bowen [14], [19] and by M. E. GURTIN [22], [23]. In our paper we adopt the definitions formerly proposed by BowEN, noting that the ones given by GURTIN are somewhat weaker than ours; anyhow this slight difference has no meaningfull consequence on our main results. As far as the "stability" of the equilibrium states is concerned we tacitly follow the assumptions laid down by CoLFMAN and GURTIN in ref. [11]. 
the inequality $(5.13)$ reads

$$
\Phi(\theta, \boldsymbol{g}, \boldsymbol{E}, \boldsymbol{H}, \boldsymbol{W}) \leqslant 0 \quad \forall(\theta, \boldsymbol{g}, \boldsymbol{E}, \boldsymbol{H}, \boldsymbol{W}) \in \mathcal{A} \subset(R-\{0\}) \times \vartheta_{3} \times \vartheta_{3} \times \mathcal{U}_{3} \times 3
$$

i) Dexinition 1. - We say that a point $\boldsymbol{x} \in \mathcal{R}$ is in equitibrium at a time $t \in(-\infty, \infty)$ if $\boldsymbol{g}(\boldsymbol{x}, t)=\mathbf{0}, \boldsymbol{E}(\boldsymbol{x}, t)=\mathbf{0}$, and there exists a temperature field $\theta=\theta^{\prime}\left(\boldsymbol{x}^{\prime}, t^{\prime}\right)$, a magnetic intensity field $\boldsymbol{H}=\boldsymbol{H}^{\prime}\left(\boldsymbol{x}^{\prime}, t^{\prime}\right)$, an extent of reaction vector $W=W^{\prime}\left(x^{\prime}, t^{\prime}\right)$-with $\left(x^{\prime}, t^{\prime}\right) \in \Re \times(-\infty, \infty)$-such that the reaction vector vanishes at $(\boldsymbol{x}, t)$; i.e.

$$
\hat{f}\left[\theta^{\prime}(\boldsymbol{x}, t), \mathbf{0}, \mathbf{0}, \boldsymbol{H}^{\prime}(\boldsymbol{x}, t), \boldsymbol{W}^{\prime}(\boldsymbol{x}, t)\right]=\mathbf{0}
$$

The collection of fields $\left(\theta^{\prime}, \mathbf{0}, \mathbf{0}, \boldsymbol{H}^{\prime}, \boldsymbol{W}^{\prime}\right)$ is called an equilibrium state for the mixture and its existence is ensured by the 1-st assumption on the rate equation (4.17).

If we take the above definition into account, the r.h.s. of (6.2) attains its maximum value at $\left(\theta^{\prime}, \mathbf{0}, \mathbf{0}, \boldsymbol{H}^{\prime}, \boldsymbol{W}^{\prime}\right)$, namely

$$
\Phi\left(\theta^{\prime}, \mathbf{0}, \mathbf{0}, \boldsymbol{H}^{\prime}, \boldsymbol{W}^{\prime}\right)=\max _{(\theta, \mathbf{g}, \boldsymbol{E}, \boldsymbol{H}, \boldsymbol{W}) \in \mathcal{A}} \Phi(\theta, \boldsymbol{g}, \boldsymbol{E}, \boldsymbol{H}, \boldsymbol{W})
$$

Therefore the well known conditions

$$
\left[\frac{d}{d \lambda} \Phi\left(\theta+\lambda a, \lambda \boldsymbol{b}, \lambda \boldsymbol{c}, \boldsymbol{H}^{\prime}+\lambda \boldsymbol{d}, \boldsymbol{W}^{\prime}+\lambda \boldsymbol{U}\right)\right]_{\lambda=0}=0
$$

and

$$
\left[\frac{\partial^{2}}{d \lambda^{2}} \Phi\left(\theta+\lambda a, \lambda \boldsymbol{b}, \lambda \boldsymbol{c}, \boldsymbol{H}^{\prime}+\lambda \boldsymbol{d}, \boldsymbol{W}^{\prime}+\lambda \boldsymbol{U}\right)\right]_{\lambda=0} \leqslant 0
$$

must hold for all $(a, b, c, d, U) \in \mathcal{A}$.

A straightforward, though rather tedious, calculation enables us to arrive at the following theorem $\left({ }^{2}\right)$.

THEOREM 6. - In an equilibrium state, the response functions of the constitutive relations (4.16) must, necessarily, be such that

$$
\begin{aligned}
& \partial_{\theta} \hat{\boldsymbol{f}}\left(\theta^{\prime}, \mathbf{0}, \mathbf{0}, \boldsymbol{H}^{\prime}, W^{\prime}\right) * \hat{\alpha}_{\mathrm{em}}\left(\theta^{\prime}, \mathbf{0}, \boldsymbol{H}^{\prime}, W^{\prime}\right)=0, \\
& {\left[\partial_{\boldsymbol{H}} \hat{\boldsymbol{f}}\left(\theta^{\prime}, \mathbf{0}, \mathbf{0}, \boldsymbol{H}^{\prime}, \boldsymbol{W}^{\prime}\right)\right] \hat{\alpha}_{\mathrm{em}}\left(\theta^{\prime}, \mathbf{0}, \boldsymbol{H}^{\prime}, \boldsymbol{W}^{\prime}\right)=\mathbf{0},} \\
& {\left[\nabla_{\boldsymbol{W}} \hat{\boldsymbol{f}}\left(\theta^{\prime}, \mathbf{0}, \mathbf{0}, \boldsymbol{H}^{\prime}, \boldsymbol{W}^{\prime}\right)\right]^{T} \hat{\boldsymbol{\alpha}}_{\mathrm{em}}\left(\theta^{\prime}, \mathbf{0}, \boldsymbol{H}^{\prime}, \boldsymbol{W}^{\prime}\right)=\mathbf{0}}
\end{aligned}
$$

$\left({ }^{2}\right)$ For the sake of simplicity, an analysis of the consequences of $(6.6)$ is not carried out because of the formal complications it would entail. 
and

$$
\begin{aligned}
& \hat{\boldsymbol{q}}\left(\theta^{\prime}, \mathbf{0}, \mathbf{0}, \boldsymbol{H}^{\prime}, W^{\prime}\right)=-\theta^{\prime}\left[\partial_{g} \hat{f}\left(\theta^{\prime}, \mathbf{0}, \mathbf{0}, \boldsymbol{H}^{\prime}, W^{\prime}\right)\right] \hat{\alpha}_{\mathrm{em}}\left(\theta^{\prime}, \mathbf{0}, \boldsymbol{H}^{\prime}, \boldsymbol{W}^{\prime}\right), \\
& \hat{\boldsymbol{j}}\left(\theta^{\prime}, \mathbf{0}, \mathbf{0}, \boldsymbol{H}^{\prime}, \boldsymbol{W}^{\prime}\right)=\quad\left[\partial_{\boldsymbol{E}} \hat{\boldsymbol{f}}\left(\theta^{\prime}, \mathbf{0}, \mathbf{0}, \boldsymbol{H}^{\prime}, \boldsymbol{W}^{\prime}\right)\right] \hat{\alpha}_{\mathrm{em}}\left(\theta^{\prime}, \mathbf{0}, \boldsymbol{H}, \boldsymbol{W}^{\prime}\right),
\end{aligned}
$$

where $\partial_{\boldsymbol{g}} \hat{\boldsymbol{f}}, \partial_{\boldsymbol{E}} \hat{\boldsymbol{f}}, \partial_{\boldsymbol{H}} \hat{\boldsymbol{f}} \in \mathcal{L}\left(\mathcal{S} \cap \mathcal{G}, \bigcup_{3}\right)$ and $\nabla_{\boldsymbol{W}} \hat{\boldsymbol{f}} \in \mathcal{L}(\mathcal{S} \cap \mathcal{G})$.

We notice at once from (6.8) that an equilibrium state does not imply both heat flux and electric current to vanish. Moreover, by (6.7), the electromagnetic chemical affinity is restricted but it is not necessarily zero and this restriction may be useful interpreted within the more abstract framework introduced in the 3-rd section

The first of (6.7) can be regarded as a condition of orthogonality between the two vectors $\hat{\alpha}_{\text {em }}$ and $\hat{\partial}_{\theta} \hat{f}$ (both belonging to the restricted reaction space $\delta \cap \mathfrak{G}$ ), when evaluated in a state of equilibrium; i.e.

$$
\hat{\boldsymbol{\alpha}}_{\in \mathrm{m}} \perp \partial_{\theta} \hat{\boldsymbol{f}}
$$

at $\left(\theta^{\prime}, \mathbf{0}, \mathbf{0}, \boldsymbol{H}^{\prime}, W^{\prime}\right)$.

Recalling that the kernels of the two linear transformations

$$
\partial_{H} \hat{f}: S \cap \mathscr{C} \rightarrow \bigcup_{3} \quad \text { and } \quad\left(\nabla_{W} \hat{f}\right)^{T}: s \cap \mathscr{C} \rightarrow S \cap \mathscr{C}
$$

are given by

$$
\mathcal{N}\left[\left(\partial_{\boldsymbol{H}} \hat{f}\right)\right] \equiv\left\{\boldsymbol{y} \in \operatorname{S\cap } \mathcal{C}:\left(\partial_{\boldsymbol{H}} \hat{f}\right) \boldsymbol{y}=\mathbf{0}\right\}
$$

and

$$
\mathcal{N}\left[\left(\nabla_{W} \hat{\boldsymbol{f}}\right)^{T}\right] \equiv\left\{\boldsymbol{y} \in \mathcal{S} \cap \mathcal{G}:\left(\nabla_{W} \hat{\boldsymbol{f}}\right)^{T} \boldsymbol{y}=\mathbf{0}\right\}
$$

respectively, eqs. $(6.7)_{1},(6.7)_{2}$ become

$$
\hat{\alpha}_{\mathrm{em}} \in \mathcal{N}\left[\left(\partial_{\boldsymbol{H}} \hat{f}\right)\right] \subset S \cap \mathcal{C}
$$

and

$$
\hat{\alpha}_{\mathrm{em}} \in \mathcal{N}\left[\left(\nabla_{W} \hat{f}\right)^{T}\right] \subset S \cap \mathcal{C}
$$

at $\left(\theta^{\prime}, \mathbf{0}, \mathbf{0}, \boldsymbol{H}^{\prime}, \boldsymbol{W}^{\prime}\right)$. The simultaneous validity of $(6.9),(6.12),(6.13)$ yields the most expressive condition

$$
\partial_{\theta} \hat{f} \perp \hat{\alpha}_{\mathrm{em}} \in \mathcal{N}\left[\left(\partial_{\boldsymbol{H}} \hat{f}\right)\right] \cap \mathcal{N}\left[\left(\nabla_{\boldsymbol{W}} \hat{f}\right)^{T}\right]
$$

at $\left(\theta^{\prime}, \mathbf{0}, \mathbf{0}, \boldsymbol{H}^{\prime}, \boldsymbol{W}^{\prime}\right)$; thus we can say: in a state of equilibrium the electromagnetic chemical affinity vector $\hat{\alpha}_{\mathrm{em}}$ must be orthogonal to the vector $\partial_{\theta} \hat{f}$ and must belong to the linear subspace of $\delta \cap \mathcal{C}$ that is the intersection of the liernets of the two linear transformations $\partial_{\boldsymbol{H}} \hat{\boldsymbol{f}},\left(\nabla_{\boldsymbol{W}} \hat{\boldsymbol{f}}\right)^{T}$. 
REMARK 1. - In many common situations it is usual to assume the regularity of the linear transformation $\left(\nabla_{W} \hat{f}\right)^{T}$ in the state of equilibrium. More precisely, if $\operatorname{det}\left\|\left(\nabla_{W} \hat{\boldsymbol{f}}\right)^{T}\right\| \equiv \operatorname{det}\left\|\left(\nabla_{W} \hat{\boldsymbol{f}}\right)\right\| \neq 0$ at equilibrium then $\nabla_{W} \hat{\boldsymbol{f}}$ belongs to the group of all the endomorphisms of $S \cap \mathscr{G}$; i.e.

$$
\nabla_{W} \hat{f} \in \mathfrak{L}^{*}(\mathcal{S} \cap \mathcal{G})
$$

when $(\theta, \mathbf{g}, \boldsymbol{E}, \boldsymbol{H}, \boldsymbol{W}) \equiv\left(\theta^{\prime}, \mathbf{0}, \mathbf{0}, \boldsymbol{H}^{\prime}, \boldsymbol{W}^{\prime}\right)$. In this case, a simple theorem of linear algebra [6] gives

$$
\mathcal{N}\left[\left(\nabla_{\boldsymbol{W}} \hat{\boldsymbol{f}}\right)^{T}\right] \equiv\{\mathbf{0}\}
$$

at $\left(\theta^{\prime}, \mathbf{0}, \mathbf{0}, \boldsymbol{H}^{\prime}, \boldsymbol{W}^{\prime}\right)$; whence by (6.14), we find

$$
\hat{\boldsymbol{\alpha}}_{\mathrm{em}}\left(\theta^{\prime}, \mathbf{0}, \boldsymbol{H}^{\prime}, \boldsymbol{W}^{\prime}\right)=\mathbf{0} \text {. }
$$

Thus, the electromagnetic ohemical affinity vector vanishes in an equitibrium state if the Tinear transformation $\left(\nabla_{\mathbb{W}} \hat{f}\right)^{T}$ is regular in this state.

ii) We see that, also in the presence of electromagnetic fields, the previous definition of an equilibrium state is not fully satisfactory from the physico-chemical point of view, if we do not introduce additional assumptions. Adopting the spirit of Bowen's works [14], [19], we question now whether or not the vanishing of the electromagnetic chemical affinity implies anything about the reaction vector; in this connection, we lay down the following new definition.

DEFINITION 2. - We say that a point $\boldsymbol{x} \in \mathcal{R}$ is in thermal equitibrium at a time $t \in(-\infty, \infty)$ if $\boldsymbol{g}(x, t)=\mathbf{0}, \boldsymbol{E}(\boldsymbol{x}, t)=\mathbf{0}$ and there exists a temperature field $\theta=\theta^{\prime \prime}\left(\boldsymbol{x}^{\prime \prime}, t^{\prime \prime}\right)$, a magnetic intensity field $\boldsymbol{H}=\boldsymbol{H}^{\prime \prime}\left(\boldsymbol{x}^{\prime \prime}, t^{\prime \prime}\right)$, an extent of reaction vector $\boldsymbol{W}=\boldsymbol{W}^{\prime \prime}\left(\boldsymbol{x}^{\prime \prime}, t^{\prime \prime}\right)$ - with $\left(\boldsymbol{x}^{\prime \prime}, t^{\prime \prime}\right) \in \mathcal{R} \times(-\infty, \infty)$ - such that the electromagnetic chemical affinity vanishes at $(x, t)$; i.e.

$$
\hat{\alpha}_{\mathrm{em}}\left[\theta^{\prime \prime}(\boldsymbol{x}, t), \mathbf{0}, \boldsymbol{H}^{\prime \prime}(\boldsymbol{x}, t), \boldsymbol{W}^{\prime \prime}(\boldsymbol{x}, t)\right]=\mathbf{0} .
$$

The collection of fields $\left(\theta^{\prime \prime}, \mathbf{0}, \mathbf{0}, \boldsymbol{H}^{\prime \prime}, \boldsymbol{W}^{\prime \prime}\right)$ is called a thermal equitibrium state and its existence is assumed as a consequence of a suitable hypothesis on the response function $\hat{\xi}$ for the Gibbs free enthalpy density.

Also taking this definition into account, the r.h.s. of (6.2) attains its maximum value at $\left(\theta^{\prime \prime}, \mathbf{0}, \mathbf{0}, \boldsymbol{H}^{\prime \prime}, W^{\prime \prime}\right)$, i.e.

$$
\Phi\left(\theta^{\prime \prime}, \mathbf{0}, \mathbf{0}, \boldsymbol{H}^{\prime \prime}, \mathbb{W}^{\prime \prime}\right)=\max _{(\theta, \boldsymbol{g}, \boldsymbol{E}, \boldsymbol{B}, \boldsymbol{W}) \in \boldsymbol{A}} \Phi(\theta, \boldsymbol{g}, \boldsymbol{E}, \boldsymbol{H}, \boldsymbol{W}) .
$$

Obviously we can follow a procedure, analogous to the one outlined in the first part of this section, whose most interesting results are summarized in the following theorem. 
THEOREM 7. - In a thermal equilibrium state, the response functions of the constitutive relations (4.16) must necessarily satisfy the conditions

$$
\begin{aligned}
& \partial_{\theta} \hat{\boldsymbol{\alpha}}_{\mathrm{em}}\left(\theta^{\prime \prime}, \mathbf{0}, \boldsymbol{H}^{\prime \prime}, \boldsymbol{W}^{\prime \prime}\right) * \hat{\boldsymbol{f}}\left(\theta^{\prime \prime}, \mathbf{0}, \mathbf{0}, \boldsymbol{H}^{\prime \prime}, \boldsymbol{W}^{\prime \prime}\right)=\mathbf{0}, \\
& {\left[\hat{\partial}_{\boldsymbol{H}} \hat{\boldsymbol{\alpha}}_{\mathrm{em}}\left(\theta^{\prime \prime}, \mathbf{0}, \boldsymbol{H}^{\prime \prime}, \boldsymbol{W}^{\prime \prime}\right)\right] \hat{\boldsymbol{f}}\left(\theta^{\prime \prime}, \mathbf{0}, \mathbf{0}, \boldsymbol{H}^{\prime \prime}, W^{\prime \prime}\right)=\mathbf{0},} \\
& {\left[\nabla_{\boldsymbol{W}} \hat{\boldsymbol{\alpha}}_{\mathrm{em}}\left(\theta^{\prime \prime}, \mathbf{0}, \boldsymbol{H}^{\prime \prime}, \boldsymbol{W}^{\prime \prime}\right)\right] \hat{\boldsymbol{f}}\left(\theta^{\prime \prime}, \mathbf{0}, \mathbf{0}, \boldsymbol{H}^{\prime \prime}, \boldsymbol{W}^{\prime \prime}\right)=\mathbf{0}}
\end{aligned}
$$

and

$$
\begin{aligned}
& \hat{\mathbf{q}}\left(\theta^{\prime \prime}, \mathbf{0}, \mathbf{0}, \boldsymbol{H}^{\prime \prime}, \boldsymbol{W}^{\prime \prime}\right)=\mathbf{0} \\
& \hat{\boldsymbol{j}}\left(\theta^{\prime \prime}, \mathbf{0}, \mathbf{0}, \boldsymbol{H}^{\prime \prime}, \boldsymbol{W}^{\prime \prime}\right)=\left[\partial_{\mathbf{E}} \hat{\boldsymbol{\alpha}}_{\mathrm{em}}\left(\theta^{\prime \prime}, \mathbf{0}, \boldsymbol{H}^{\prime \prime}, W^{\prime \prime}\right)\right] \hat{\boldsymbol{f}}\left(\theta^{\prime \prime}, \mathbf{0}, \mathbf{0}, \boldsymbol{H}^{\prime \prime}, \boldsymbol{W}^{\prime \prime}\right)
\end{aligned}
$$

where $\partial_{\boldsymbol{E}} \hat{\alpha}_{\mathrm{em}}, \partial_{\boldsymbol{H}} \hat{\alpha}_{\mathrm{em}} \in \mathcal{L}\left(\delta \cap \mathcal{G}, \mathcal{V}_{3}\right), \nabla_{W} \hat{\alpha}_{\mathrm{em}} \equiv\left(\nabla_{\boldsymbol{W}} \hat{\alpha}_{\mathrm{em}}\right)^{T} \in \mathcal{L}(\mathcal{S} \cap \mathcal{G})\left(^{3}\right)$.

The results espressed by (6.20) are to regarded as stronger than those given by $(6.8)$; in fact, even if the electric current is not zero the heat flux vanishes in a state of thermal equilibrium $\left({ }^{4}\right)$.

Also in this case, the restrictions (6.19), now imposed on the reaction vector $\hat{f}$ in a state of thermal equilibrium, may be seen as "geometrical " properties of $\hat{f}$ in the restricted reaction space $S \cap \mathcal{G}$. Adopting again definitions and methods, already employed in the first part of this section, we can easily show that (6.19) are equivalent to

$$
\partial_{\theta} \hat{\alpha}_{\mathrm{em}} \perp \hat{f} \in \mathcal{N}\left[\left(\partial_{\boldsymbol{H}} \hat{\alpha}_{\mathrm{em}}\right)\right] \cap \mathcal{N}\left[\left(\nabla_{\boldsymbol{W}} \hat{\alpha}_{\mathrm{em}}\right)\right]
$$

at $\left(\theta^{\prime \prime}, \mathbf{0}, \mathbf{0}, \boldsymbol{H}^{\prime \prime}, \boldsymbol{W}^{\prime \prime}\right)$. This means that in a state of thermal equilibrium the reaction vector $\hat{f}$ must be orthogonal to the vector $\partial_{\theta} \hat{\alpha}_{\mathrm{em}}$ and must belong to the linear subspace of $S \cap \mathcal{G}$ that is the intersection of the kernets of the linear transformations $\partial_{\boldsymbol{H}} \hat{\alpha}_{\mathrm{em}}$, $\nabla_{W} \hat{\alpha}_{\mathrm{em}}$.

REMARK 2. - Let us assume that $\nabla_{\boldsymbol{W}} \hat{\alpha}_{\mathrm{em}} \equiv \boldsymbol{H}_{\mathfrak{w}}(\hat{\xi})$ is regular in a state of thermal equilibrium; this implies that the hessian determinant of $\hat{\xi}$ with respect to $W$ is not zero in such a state, or, equivalentely,

$$
\nabla_{W} \hat{\alpha}_{\mathrm{em}} \equiv \boldsymbol{H}_{W}(\hat{\xi}) \in \mathfrak{L}^{*}(\delta \cap \zeta)
$$

at $\left(\theta^{\prime \prime}, \mathbf{0}, \mathbf{0}, \boldsymbol{H}^{\prime \prime}, \boldsymbol{W}^{\prime \prime}\right)$. Then, a previously quoted theorem of linear algebra gives

$\left.{ }^{3}\right)$ Recalling def. (5.7) for $\hat{\alpha}_{e m}$, we have $\nabla_{W} \hat{\alpha}_{e m}=\boldsymbol{H}_{W}(\hat{\xi})$, where $\boldsymbol{H}_{W}(\xi)$ denotes the symzymetric hessian tensor of $\xi$ with respect to $\boldsymbol{W}$; moreover $\partial_{\boldsymbol{E}} \hat{\alpha}_{\mathrm{em}}=-\nabla_{\boldsymbol{W}} \hat{\boldsymbol{D}}$ and $\partial_{\boldsymbol{H}} \hat{\boldsymbol{x}}_{\mathrm{em}}=-\nabla_{\boldsymbol{W}} \hat{\boldsymbol{B}}$ are immediate consequences of $(5.12)$ and $(5.12)_{3}$ respectively.

(4) This remark justifies the denomination "thermal " adopted for defining this type of equilibrium. 
$\mathcal{N}\left[\left(\nabla \hat{\boldsymbol{\alpha}}_{\mathrm{em}}\right)\right] \equiv \mathcal{N}\left[\boldsymbol{H}_{\mathbb{W}}(\xi)\right] \equiv\{\boldsymbol{0}\}$ at $\left(\theta^{\prime \prime}, \mathbf{0}, \mathbf{0}, \boldsymbol{H}^{\prime \prime}, \boldsymbol{W}^{\prime \prime}\right)$, whence

$$
\hat{\boldsymbol{f}}\left(\theta^{\prime \prime}, \mathbf{0}, \mathbf{0}, \boldsymbol{H}^{\prime \prime}, \boldsymbol{W}^{\prime \prime}\right)=0
$$

as a consequence of $(6.21)$.

In conclusion, the reaction vector vanishes in a thermal equilibrium state if the linear transformation $\nabla_{W} \hat{\alpha}_{\mathrm{em}} \equiv \boldsymbol{H}_{W}(\hat{\xi})$ is regular in this state.

iii) At this point we are able to give the definition of "classical equilibrium " (i.e. that kind of equilibrium commonly accepted by all the authors) and to see how it is related to the previous definitions of equilibrium and of thermal equilibrium.

Definition 3. - We say that a point $x \in \mathfrak{R}$ is in classical equilibrium at a time $t \in(-\infty, \infty)$ if $\boldsymbol{g}(\boldsymbol{x}, t)=\mathbf{0}, \boldsymbol{E}(\boldsymbol{x}, t)=\mathbf{0}$, and there exists a temperature field $\theta=\tilde{\theta}(\tilde{\boldsymbol{x}}, \tilde{t})$, a magnetic intensity field $\boldsymbol{H}=\tilde{\boldsymbol{\mu}}(\tilde{\boldsymbol{x}}, \tilde{t})$, an extent of reaction vector $\tilde{\boldsymbol{W}}=\tilde{\boldsymbol{W}}(\tilde{\boldsymbol{x}}, \tilde{t})$ -with $(\tilde{x}, \tilde{t}) \in \mathcal{R} \times(-\infty, \infty)$-such that both the reaction vector and the electromagnetic chemical affinity vector vanish at $(x, t)$; i.e.

$$
\begin{aligned}
& \hat{f}[\tilde{\theta}(\boldsymbol{x}, t), \mathbf{0}, \mathbf{0}, \tilde{\boldsymbol{H}}(\boldsymbol{x}, t), \tilde{\boldsymbol{W}}(\boldsymbol{x}, t)]=\mathbf{0} \\
& \hat{\boldsymbol{\alpha}}_{\mathrm{em}}[\tilde{\theta}(\boldsymbol{x}, t), \mathbf{0}, \quad \tilde{\boldsymbol{H}}(\boldsymbol{x}, t), \tilde{\boldsymbol{W}}(\boldsymbol{x}, t)]=\mathbf{0} .
\end{aligned}
$$

The set $(\tilde{\theta}, \mathbf{0}, \mathbf{0}, \tilde{\boldsymbol{H}}, \tilde{\boldsymbol{W}})$, which is an equilibrium and a thermal equilibrium state at the same time, is called a classical equitibrium state. Obviously in such a state we have the desired results:

$$
\hat{\boldsymbol{q}}(\tilde{\theta}, \mathbf{0}, \mathbf{0}, \tilde{\boldsymbol{H}}, \widetilde{\boldsymbol{W}})=\mathbf{0}, \quad \hat{\boldsymbol{j}}(\tilde{\theta}, \mathbf{0}, \mathbf{0}, \tilde{\boldsymbol{H}}, \widetilde{\boldsymbol{W}})=\mathbf{0}
$$

If we recall now the remarks made at the end of the 6 -th and of the 7 -th theorems of this section, we can state the following immediate consequences.

Corollary 1. - If the linear transformation $\nabla_{\mathbb{F}} \hat{f}: \delta \cap \mathfrak{G} \rightarrow \delta \cap \mathfrak{G}$ is regular -i.e. if $\nabla_{W} \hat{f} \in \mathfrak{L}^{*}(\mathcal{S} \cap \mathcal{G})$-at an equilibrium state, then this state is a elassical equilibrium state.

Corollary 2. - If the linear transformation $\nabla_{\mathbb{W}} \hat{\alpha}_{\mathrm{em}}: \delta \cap \mathfrak{G} \rightarrow \delta \cap \mathcal{C}$ is regular -i.e. if $\nabla_{W} \hat{\alpha}_{\mathrm{em}} \in \mathcal{L}^{*}(S \cap \mathcal{E})$-at a thermal equilibrium state, then this state is a classical equilibrium state.

CoRoLlaRY 3. - If the two linear transformations $\nabla_{W} \hat{f}, \nabla_{W} \hat{\alpha}_{\mathrm{em}}: S \cap \mathfrak{C} \rightarrow S \cap \mathfrak{C}$ are regular-i.e. if $\nabla_{\boldsymbol{W}} \hat{\boldsymbol{f}}, \nabla_{\mathbb{W}} \hat{a}_{\mathrm{em}} \in \mathfrak{L}^{*}(\delta \cap \mathcal{G})$-at a state $(\theta, \mathbf{0}, \mathbf{0}, \boldsymbol{H}, \boldsymbol{W})$, then the equilibrium and the thermal equilibrium states are equivalent and coincide with the classical eqnilibrium state; namely

$$
\hat{f}(\theta, \mathbf{0}, \mathbf{0}, \boldsymbol{H}, W)=\mathbf{0} \Leftrightarrow \hat{\alpha}_{\mathrm{epm}}(\theta, \mathbf{0}, \boldsymbol{H}, W)=\mathbf{0},
$$


REMARK 3. - The assumption $\hat{\boldsymbol{f}} \in \mathrm{C}^{\infty}(\mathfrak{A})$ and the regularity condition $\operatorname{det}\left\|\nabla_{\boldsymbol{w}} \hat{\boldsymbol{f}}\right\| \neq 0$ in a state of equilibrium $\left(\theta^{\prime}, \mathbf{0}, \mathbf{0}, \boldsymbol{H}^{\prime}, W^{\prime}\right)$ ensure the existence of a neighborhood $\mathfrak{J}^{\prime}$ of $\left(\theta^{\prime}, \boldsymbol{H}^{\prime}\right)$ and of a $\mathcal{C}^{1}$ vector valued function $\overline{\boldsymbol{W}}^{\prime}: \mathfrak{J}^{\prime} \rightarrow \mathfrak{3}$ such that

$$
\boldsymbol{W}^{\prime}=\overline{\boldsymbol{W}}^{\prime}(\theta, \boldsymbol{H}) \quad \forall(\theta, \boldsymbol{H}) \in J^{\prime}
$$

and

$$
\hat{f}\left[\theta, \mathbf{0}, \mathbf{0}, \boldsymbol{H}, \overline{\boldsymbol{W}}^{\prime}(\theta, \boldsymbol{H})\right]=\mathbf{0} \quad \forall(\theta, \boldsymbol{H}) \in \mathfrak{J}^{\prime}
$$

Then the relations $(6.7),(6.8)$ of the 6 -th theorem give

$$
\begin{array}{rc}
\hat{\boldsymbol{\alpha}}_{\mathrm{em}}\left[\theta, \mathbf{0}, \mathbf{0}, \boldsymbol{H}, \bar{W}^{\prime}(\theta, \boldsymbol{H})\right]=\mathbf{0} & (\theta, \boldsymbol{H}) \in \mathcal{J}^{\prime} \\
\hat{\boldsymbol{q}}\left[\theta, \mathbf{0}, \mathbf{0}, \boldsymbol{H}, \bar{W}^{\prime}(\theta, \boldsymbol{H})\right]=\mathbf{0} & (\theta, \boldsymbol{H}) \in \mathfrak{J}^{\prime} \\
\hat{\boldsymbol{j}}\left[\theta, \mathbf{0}, \mathbf{0}, \boldsymbol{H}, \bar{W}^{\prime}(\theta, \boldsymbol{H})\right]=\mathbf{0} & (\theta, \boldsymbol{H}) \in \mathfrak{J}^{\prime}
\end{array}
$$

In a similar way the smoothness condition $\hat{\xi} \in \mathbb{C}^{\infty}(\mathfrak{A})$, together with $\operatorname{det}\left\|\boldsymbol{H}_{W}(\hat{\xi})\right\| \neq 0$ in a state of thermal equilibrium, implies the existence of a neighborhood $J^{\prime \prime}$ of $\left(\theta^{\prime \prime}, \boldsymbol{H}^{\prime \prime}\right)$ and of a $\mathrm{C}^{1}$ function $\overline{\boldsymbol{W}}^{\prime \prime}: \mathfrak{J}^{\prime \prime} \rightarrow 3$ such that

$$
\boldsymbol{W}^{\prime \prime}=\overline{\boldsymbol{W}}^{\prime \prime}(\theta, \boldsymbol{H}) \quad \forall(\theta, \boldsymbol{H}) \in \mathfrak{J}^{\prime \prime}
$$

and

$$
\hat{\boldsymbol{\alpha}}_{\mathrm{gm}}\left[\theta, \mathbf{0}, \boldsymbol{H}, \overline{\boldsymbol{W}}^{\prime \prime}(\theta, \boldsymbol{H})\right]=\mathbf{0} \quad \forall(\theta, \boldsymbol{H}) \in J^{\prime \prime}
$$

By $(6.30),(6.31)$, the relations $(6.19),(6.20)$ yield

$$
\begin{aligned}
\hat{\boldsymbol{f}}\left[\theta, \mathbf{0}, \mathbf{0}, \boldsymbol{H}, \overline{\boldsymbol{W}}^{\prime \prime}(\theta, \boldsymbol{H})\right]=\mathbf{0} & (\theta, \boldsymbol{H}) \in \mathfrak{J}^{\prime \prime} \\
\hat{\boldsymbol{q}}\left[\theta, \mathbf{0}, \mathbf{0}, \boldsymbol{H}, \overline{\boldsymbol{W}}^{\prime \prime}(\theta, \boldsymbol{H})\right]=\mathbf{0} & (\theta, \boldsymbol{H}) \in \mathfrak{J}^{\prime \prime}, \\
\hat{\boldsymbol{j}}\left[\theta, \mathbf{0}, \mathbf{0}, \boldsymbol{H}, \overline{\boldsymbol{W}}^{\prime \prime}(\theta, \boldsymbol{H})\right]=\mathbf{0} & (\theta, \boldsymbol{H}) \in \mathfrak{J}^{\prime \prime}
\end{aligned}
$$

Finally, when $\hat{\xi}, \hat{f} \in \mathrm{C}^{\infty}(\mathcal{A})$ and the conditions of the 3-rd corollary are satisfied (i.e. we are in a classical equilibrium state), we can conclude that there exist a $\mathrm{C}^{1}$ function $\tilde{\boldsymbol{W}}^{\dagger}: \mathfrak{J}^{\prime} \cap \mathfrak{J}^{\prime \prime} \neq \phi \rightarrow 3$ such that

$$
\tilde{\boldsymbol{W}}=\tilde{\boldsymbol{W}}^{\dagger}(\theta, \boldsymbol{H}) \quad \forall(\theta, \boldsymbol{H}) \in J^{\prime} \cap J^{\prime \prime}
$$

and

$$
\hat{\boldsymbol{f}}\left[\theta, \mathbf{0}, \mathbf{0}, \boldsymbol{H}, \tilde{\boldsymbol{W}}^{\dagger}(\theta, \boldsymbol{H})\right]=\mathbf{0} \quad \forall(\theta, \boldsymbol{H}) \in \mathfrak{J}^{\prime} \cap \mathfrak{J}^{\prime \prime},
$$

$$
\hat{\boldsymbol{\alpha}}_{\mathrm{em}}\left[\theta, \mathbf{0}, \boldsymbol{H}, \tilde{\boldsymbol{W}}^{\dagger}(\theta, \boldsymbol{H})\right]=\mathbf{0} \quad \forall(\theta, \boldsymbol{H}) \in \mathfrak{J}^{\prime} \cap J^{\prime \prime} .
$$


In this case the eqs. (6.25) can be rewritten in the following way

$$
\begin{array}{cc}
\hat{\boldsymbol{q}}\left[\theta, \mathbf{0}, \mathbf{0}, \boldsymbol{H}, \tilde{\boldsymbol{W}}^{\dagger}(\theta, \boldsymbol{H})\right]=\mathbf{0} & (\theta, \boldsymbol{H}) \in \mathfrak{J}^{\prime} \cap \mathfrak{J}^{\prime \prime} \\
\hat{\mathfrak{j}}\left[\theta, \mathbf{0}, \mathbf{0}, \boldsymbol{H}, \tilde{\boldsymbol{W}}^{\dagger}(\theta, \boldsymbol{H})\right]=\mathbf{0} & (\theta, \boldsymbol{H}) \in \mathfrak{J}^{\prime} \cap \mathfrak{J}^{\prime \prime}
\end{array}
$$

The results $(6.29)_{2,3},(6.32)_{2,3}$ and (6.35) allow us to conclude that, under generally accepted smoothness conditions for some response functions and regularity assumption for some linear transformations, the heat flux and the electrie current vanish, i.e.

$$
\boldsymbol{q}=\widehat{\mathbf{q}}(\theta, \mathbf{0}, \mathbf{0}, \boldsymbol{H})=\mathbf{0} \quad \text { and } \quad \boldsymbol{j}=\widehat{\boldsymbol{j}}(\theta, \mathbf{0}, \mathbf{0}, \boldsymbol{H})=\mathbf{0}
$$

where the domains and the nature of the vector valued functions $\widehat{\boldsymbol{q}}$ and $\hat{\boldsymbol{j}}$ are different according to the various types of equilibrium taken into consideration.

\section{7. - Thermal and electric conduction « near » a classical equilibrium state.}

The third remark at the end of the previous section, with the main result embodied by eqs. (6.36), allows us to express the heat flux $\boldsymbol{q}$ and the electric current $j$ by means of meaningful 1-st-order expansions. More precisely we can write $\boldsymbol{q}$ and $\boldsymbol{j}$ in a neighborhood either of an equilibrium state or of a thermal equilibrium state or of a classical equilibrium state, provided each state be endowed with suitable smoothness and regularity conditions.

For the sake of clarity, let us consider a classical equitibrium state $(\tilde{\theta}, \mathbf{0}, \mathbf{0}, \tilde{\boldsymbol{H}}, \widetilde{\boldsymbol{W}})$; then, by (6.33), (6.34) and (6.35), we have

$$
\tilde{\boldsymbol{q}}(\tilde{\theta}, \mathbf{0}, \mathbf{0}, \tilde{\boldsymbol{H}}) \equiv \mathbf{0} \quad(\theta, \boldsymbol{H}) \in \tilde{\mathfrak{J}} \quad \text { and } \quad \tilde{\boldsymbol{j}}(\tilde{\theta}, \mathbf{0}, \mathbf{0}, \tilde{\boldsymbol{H}}) \equiv \mathbf{0} \quad(\theta, \boldsymbol{H}) \in \tilde{\mathfrak{J}}
$$

where $\tilde{J}$ is a neighborhood of $(\tilde{\theta}, \tilde{\boldsymbol{H}})$ given by

$$
\tilde{\mathfrak{J}} \equiv\left\{(\theta, \boldsymbol{H}) \in(R-\{0\}) \times \mathcal{V}_{3}:|\theta-\tilde{\theta}|<\delta_{\theta},|\boldsymbol{H}-\boldsymbol{H}|<\delta_{\tilde{\boldsymbol{H}}}\right\}
$$

with $\delta_{\tilde{\theta}}, \delta_{\tilde{\mathbf{H}}} \in R_{+}$.

When the temperature gradient $\boldsymbol{g}$ and the electric intensity $\boldsymbol{E}$ are not zero, it is possible to express the heat flux $\tilde{\boldsymbol{q}}$ and the electric current $\tilde{\boldsymbol{j}}$-regarded as functions of $s \equiv[\theta, \boldsymbol{g}, \boldsymbol{E}, \boldsymbol{H}]$-by means of Taylor expansions in the neighborhood of $\tilde{s} \equiv[\tilde{\theta}, \mathbf{0}, \mathbf{0}, \tilde{\boldsymbol{H}}]$, provided $(\theta, \boldsymbol{H}) \in \tilde{\mathfrak{J}}$. Thus we can write

$$
\begin{aligned}
\tilde{\boldsymbol{q}}(\theta, \boldsymbol{g}, \boldsymbol{E}, \boldsymbol{H})=(\tilde{\boldsymbol{q}})_{\tilde{s}}+\left(\partial_{\theta} \tilde{\boldsymbol{q}}\right)_{\tilde{s}}(\theta-\tilde{\theta})+\left(\partial_{\boldsymbol{g}} \tilde{\boldsymbol{q}}\right)_{s} \boldsymbol{g} & +\left(\partial_{\boldsymbol{E}} \tilde{\boldsymbol{q}}\right)_{s} \boldsymbol{E}+ \\
& +\left(\partial_{\boldsymbol{H}} \tilde{\boldsymbol{q}}\right)_{\tilde{s}}(\boldsymbol{H}-\tilde{\boldsymbol{H}})+\boldsymbol{0}\left(|s-\tilde{s}|^{2}\right)
\end{aligned}
$$


and

$$
\begin{aligned}
\tilde{\boldsymbol{j}}(\theta, \mathbf{g}, \boldsymbol{E}, \boldsymbol{H})=(\tilde{\boldsymbol{j}})_{\tilde{s}}+\left(\partial_{\theta} \tilde{\boldsymbol{j}}\right)_{\tilde{s}}(\theta-\tilde{\theta})+\left(\partial_{\boldsymbol{g}} \tilde{\boldsymbol{j}}\right)_{\tilde{s}} \mathbf{g} & +\left(\partial_{\boldsymbol{E}} \tilde{\boldsymbol{j}}\right)_{\tilde{s}} \boldsymbol{E}+ \\
& +\left(\partial_{\boldsymbol{H}} \tilde{\boldsymbol{j}}\right)_{\tilde{s}}(\boldsymbol{H}-\tilde{\boldsymbol{H}})+\mathbf{0}\left(|s-\tilde{s}|^{2}\right)
\end{aligned}
$$

where $|s-\tilde{s}|=|\theta-\tilde{\theta}|+|\boldsymbol{g}|+|\boldsymbol{E}|+|\boldsymbol{H}-\tilde{\boldsymbol{H}}|$ with $(\theta, \boldsymbol{H}) \in \tilde{\mathfrak{J}}$.

If we take eqs. (7.1) into account and if we remark that

$$
\begin{aligned}
& \left(\partial_{\theta} \tilde{\boldsymbol{q}}\right)_{\tilde{s}}=\lim _{\lambda \rightarrow 0} \frac{\tilde{\boldsymbol{q}}(\tilde{\theta}+\lambda, \mathbf{0}, \mathbf{0}, \tilde{\boldsymbol{H}})-\tilde{\boldsymbol{q}}(\tilde{\theta}, \mathbf{0}, \mathbf{0}, \tilde{\boldsymbol{H}})}{\lambda} \equiv \mathbf{0}, \\
& \left(\partial_{\theta} \tilde{\boldsymbol{j}}\right)_{\tilde{s}}=\lim _{\lambda \rightarrow 0} \frac{\tilde{\boldsymbol{j}}(\tilde{\theta}+\lambda, \mathbf{0 , 0}, \tilde{\boldsymbol{H}})-\tilde{\boldsymbol{j}}(\tilde{\theta}, \mathbf{0}, \mathbf{0}, \tilde{\boldsymbol{H}})}{\lambda} \equiv \mathbf{0}
\end{aligned}
$$

together with

$$
\begin{aligned}
& \left(\partial_{\boldsymbol{H}} \tilde{\boldsymbol{q}}\right)_{\tilde{s}} \boldsymbol{v}=\lim _{\mu \rightarrow 0} \frac{\tilde{\boldsymbol{q}}(\tilde{\theta}, \mathbf{0 , 0}, \tilde{\boldsymbol{H}}+\mu \boldsymbol{v})-\tilde{\boldsymbol{q}}(\tilde{\theta}, \mathbf{0}, \mathbf{0}, \tilde{\boldsymbol{H}})}{\mu} \equiv \mathbf{0} \\
& \left(\partial_{\mathbf{H}} \tilde{\boldsymbol{j}}\right)_{\tilde{s}} \boldsymbol{v}=\lim _{\mu \rightarrow 0} \frac{\tilde{\boldsymbol{j}}(\tilde{\theta}, \mathbf{0}, \mathbf{0}, \tilde{\boldsymbol{H}}+\mu \boldsymbol{v})-\tilde{\boldsymbol{j}}(\tilde{\theta}, \mathbf{0}, \mathbf{0}, \tilde{\boldsymbol{H}})}{\mu} \equiv \mathbf{0}
\end{aligned}
$$

of reach $v$ in $\mathcal{V}_{3}$, the expansions $(7.3)_{1,2}$ become

$$
\tilde{\boldsymbol{q}}=\left(\partial_{\boldsymbol{g}} \tilde{\boldsymbol{q}}\right)_{\tilde{s}} \boldsymbol{g}+\left(\partial_{\boldsymbol{E}} \tilde{\boldsymbol{q}}\right)_{\tilde{s}} \boldsymbol{E}+\mathbf{0}\left(|s-\tilde{s}|^{2}\right)
$$

and

$$
\tilde{\boldsymbol{j}}=\left(\partial_{\boldsymbol{g}} \tilde{\boldsymbol{j}}\right)_{\tilde{s}} \boldsymbol{g}+\left(\partial_{E} \tilde{\boldsymbol{j}}\right)_{\tilde{s}} \boldsymbol{E}+\mathbf{0}\left(|s-\tilde{s}|^{2}\right)
$$

with $(\theta, \boldsymbol{H}) \in \tilde{\mathfrak{J}}\left(^{1}\right)$.

We introduce now, the "thermal conductivity", the "thermoelectric conductivity", the "electrothermal conductivity " and the "electric conductivity" in a classical equilibriam state, by setting

$$
\widetilde{\boldsymbol{K}}=\left(\partial_{\boldsymbol{g}} \tilde{\boldsymbol{q}}\right)_{\tilde{s}}, \quad \tilde{\boldsymbol{L}}=\left(\partial_{\boldsymbol{E}} \tilde{\boldsymbol{q}}\right)_{\tilde{\boldsymbol{s}}} \quad \text { and } \quad \tilde{\boldsymbol{\tau}}=\left(\partial_{\boldsymbol{g}} \tilde{\boldsymbol{j}}\right)_{\tilde{s}}, \quad \tilde{\boldsymbol{\sigma}}=\left(\partial_{\boldsymbol{E}} \tilde{\boldsymbol{j}}\right)_{\tilde{s}}
$$

respectively; obviously $\tilde{\boldsymbol{K}}, \tilde{\boldsymbol{L}}, \tilde{\boldsymbol{\tau}}, \tilde{\boldsymbol{\sigma}} \in \mathfrak{L}\left(\mathcal{V}_{3}\right)$.

By the above definitions, our results (7.6) are summarized in the following theorem.

(') An exhaustive discussion on high order expansions for $\tilde{\boldsymbol{q}}$ and $\tilde{\boldsymbol{j}}$ in a simple solid ma. terial can be found in ref. [27]. 
THEOREM 8. - The heat flux and the electric current, "near" a classical equilibrium state of the mixture, can be expressed by the expansions

$$
\tilde{\boldsymbol{q}}=\tilde{\boldsymbol{K}} \boldsymbol{g}+\tilde{\boldsymbol{L}} \boldsymbol{E}+\mathbf{0}\left(|s-\tilde{s}|^{2}\right)
$$

and

$$
\tilde{j}=\tilde{\tau} g+\tilde{\sigma} \boldsymbol{E}+\mathbf{0}\left(|s-\tilde{s}|^{2}\right)
$$

with $(\theta, \boldsymbol{H}) \in \tilde{\mathfrak{J}}$.

Thus we have proved that the formulas, which hold for a stationary rigid material with instantaneous electromagnetic response, are also valid "near" a classical equilibrium state of a non-diffusing reacting mixture of stationary rigid materials with the assumption of an instantaneous electromagnetic response of the mixture as a whole [24], [26], [27].

The four tensors (7.7), defined in a state of classical equilibrium, must be consistent with the "chemical dissipation inequality for thermal and electric conduction" (5.13) provided this inequality be considered at the same order of approximation as the expansions $(7.8)_{1,2}$.

In this connection we recall the two equations $(6.34)_{1}$ and $(6.34)_{2}$, which we rewrite in such a way:

$$
\tilde{\boldsymbol{f}}(\theta, \mathbf{0}, \mathbf{0}, \boldsymbol{H}) \equiv \mathbf{0} \quad \text { and } \quad \tilde{\alpha}_{\mathrm{em}}(\theta, \mathbf{0}, \boldsymbol{H}) \equiv \mathbf{0}
$$

when $(\theta, \boldsymbol{H}) \in \tilde{\mathfrak{J}}$.

Following the procedure already adopted for the two vector valued functions $\tilde{\boldsymbol{q}}$ and $\boldsymbol{j}$ of $\mathcal{V}_{3}$, we can express the two vector valued functions $\tilde{\boldsymbol{f}}$ and $\tilde{\boldsymbol{\alpha}}_{\mathrm{em}}$ of $s \cap \mathcal{C}$ by means of Taylor expansions. Obviously the argument of $\tilde{\boldsymbol{f}}$ is $s \equiv[\theta, \boldsymbol{g}, \boldsymbol{E}, \boldsymbol{H}]$ and the argument of $\tilde{\boldsymbol{\alpha}}_{\mathrm{em}}$ is $p \equiv[\theta, \boldsymbol{E}, \boldsymbol{H}]$, whyle the «starting " states are $\tilde{s} \equiv[\tilde{\theta}, \mathbf{0}, \mathbf{0}, \widetilde{\boldsymbol{H}}]$ and $\tilde{\boldsymbol{p}} \equiv[\tilde{\theta}, \mathbf{0}, \tilde{\boldsymbol{H}}]$ respectively. Thus we have

$$
\begin{aligned}
\tilde{\boldsymbol{f}}(\theta, \boldsymbol{g}, \boldsymbol{E}, \boldsymbol{H})=(\tilde{\boldsymbol{f}})_{\tilde{s}}+\left(\partial_{\theta} \tilde{\boldsymbol{f}}\right)_{\tilde{s}}(\theta-\tilde{\theta})+\left(\partial_{\boldsymbol{g}} \tilde{\boldsymbol{f}}\right)_{\tilde{s}}^{T} \boldsymbol{g}+ \\
+\left(\partial_{\boldsymbol{E}} \tilde{\boldsymbol{f}}\right)_{\tilde{s}}^{T} \boldsymbol{E}+\left(\partial_{\boldsymbol{H}} \tilde{\boldsymbol{f}}\right)_{\tilde{s}}^{T}(\boldsymbol{H}-\tilde{\boldsymbol{H}})+\mathbf{0}\left(|s-\tilde{s}|^{2}\right)
\end{aligned}
$$

and

$$
\begin{aligned}
\tilde{\boldsymbol{\alpha}}_{\mathrm{em}}(\theta, \boldsymbol{E}, \boldsymbol{H})=\left(\tilde{\boldsymbol{\alpha}}_{\mathrm{em}}\right)_{\tilde{p}}+\left(\partial_{\theta} \tilde{\boldsymbol{\alpha}}_{\mathrm{em}}\right)_{\tilde{p}}(\theta-\tilde{\theta}) & +\left(\partial_{\boldsymbol{E}} \tilde{\boldsymbol{\alpha}}_{\mathrm{em}}\right)_{\tilde{p}}^{T} \boldsymbol{E}+ \\
& +\left(\partial_{\boldsymbol{H}} \tilde{\boldsymbol{\alpha}}_{\mathrm{em}}\right)_{\tilde{p}}^{T}(\boldsymbol{H}-\tilde{\boldsymbol{H}})+\mathbf{0}\left(|p-\tilde{p}|^{2}\right),
\end{aligned}
$$

with $|s-\tilde{s}|=|\theta-\tilde{\theta}|+|\boldsymbol{g}|+|\boldsymbol{E}|+|\boldsymbol{H}-\tilde{\boldsymbol{H}}|$ and $|p-\tilde{p}|=|\theta-\tilde{\theta}|+|\boldsymbol{E}|+|\boldsymbol{H}-\tilde{\boldsymbol{H}}|$, provided $(\theta, \boldsymbol{H}) \in \tilde{\mathfrak{J}}$.

By eqs. (7.9), together with their immediate consequences

$$
\left(\partial_{\theta} \tilde{f}\right)_{\tilde{s}} \equiv \mathbf{0}, \quad\left(\partial_{\theta} \tilde{\alpha}_{e m}\right)_{\tilde{p}} \equiv \mathbf{0} \quad \text { and } \quad\left(\partial_{\boldsymbol{H}} \tilde{f}\right)_{\tilde{s}}^{T} \equiv \mathbf{0}, \quad\left(\partial_{\boldsymbol{H}} \tilde{\alpha}_{\mathrm{em}}\right)_{\tilde{p}}^{T} \equiv \mathbf{0}
$$


we find:

$$
\tilde{f}=\left(\partial_{g} \tilde{f}\right)_{s}^{R} \boldsymbol{g}+\left(\partial_{E} \tilde{f}\right)_{\tilde{s}}^{R} \boldsymbol{E}+\mathbf{0}\left(|s-\tilde{s}|^{2}\right)
$$

and

$$
\tilde{\alpha}_{\mathrm{em}}=\left(\partial_{E} \tilde{\alpha}_{\mathrm{em}}\right)_{\tilde{p^{\prime}}}^{T} \boldsymbol{E}+\mathbf{0}\left(|p-\tilde{p}|^{2}\right)
$$

with $(\theta, \boldsymbol{H}) \in \tilde{\mathfrak{J}}$.

Now we are able to derive the restrictions on the tensors, which are defined by eqs. (7.7) and which affect the main expansions (7.8) $)_{1.2}$, due to a suitable approximate expression of the "chemical dissipation inequality for thermal and electric conduction 》 (5.13).

In fact, when $(\theta, \boldsymbol{H}) \in \tilde{\mathfrak{J}}$, we can write

$$
\tilde{\boldsymbol{q}} \cdot \boldsymbol{g}-\tilde{\theta} \tilde{\boldsymbol{j}} \cdot \boldsymbol{E}+\tilde{\theta} \tilde{\boldsymbol{e}}_{\mathrm{em}} * \tilde{\boldsymbol{f}} \leqslant 0
$$

where $\tilde{\boldsymbol{q}}, \tilde{\boldsymbol{j}}$ and $\tilde{\boldsymbol{f}}, \tilde{\alpha}_{\mathrm{em}}$ are given by eqs. (7.8) $)_{1,2}$ and by eqs. (7.12) $)_{1,2}$ respectively.

Neglecting the higher order terms, a direct substitution yields

$$
\begin{aligned}
\tilde{\boldsymbol{K}} \boldsymbol{g} \cdot \boldsymbol{g}-\tilde{\theta} \tilde{\boldsymbol{\sigma}} \boldsymbol{E} \cdot \boldsymbol{E}+\tilde{\boldsymbol{L}} \boldsymbol{E} \cdot \boldsymbol{g}-\tilde{\theta} \tilde{\tau} \boldsymbol{g} \cdot \boldsymbol{E}+ \\
\quad+\tilde{\theta}\left(\partial_{\boldsymbol{E}} \tilde{\alpha}_{\mathrm{em}}\right)_{\tilde{p}}^{T} \boldsymbol{E} *\left(\partial_{\boldsymbol{g}} \tilde{\boldsymbol{f}}\right)_{\tilde{s}}^{T} \boldsymbol{g}+\tilde{\theta}\left(\partial_{\boldsymbol{E}} \tilde{\alpha}_{\mathrm{em}}\right)_{\tilde{p}}^{T} \boldsymbol{E} *\left(\partial_{\boldsymbol{E}} \tilde{\boldsymbol{f}}\right)_{\tilde{s}}^{T} \boldsymbol{E} \leqslant 0
\end{aligned}
$$

for each $\boldsymbol{g}$ and $\boldsymbol{E}$ in $\mathcal{V}_{3}$.

It is convenient to introduce the symmetric parts of the two tensors $\widetilde{\boldsymbol{K}}$ and $\tilde{\sigma}$, namely $\widetilde{\boldsymbol{K}}_{+}=\frac{1}{2}\left(\widetilde{\boldsymbol{K}}+\widetilde{\boldsymbol{K}}^{T}\right)$ and $\tilde{\boldsymbol{\sigma}}_{+}=\frac{1}{2}\left(\tilde{\boldsymbol{\sigma}}+\tilde{\boldsymbol{\sigma}}^{T}\right)$, and to recall the two identities:

$$
\boldsymbol{T} \boldsymbol{x} \cdot \boldsymbol{y}=\boldsymbol{x} \cdot \boldsymbol{T}^{T} \boldsymbol{y} \quad \text { and } \quad \boldsymbol{A x} * \boldsymbol{B} \boldsymbol{y}=\boldsymbol{x} \cdot \boldsymbol{A}^{T} \boldsymbol{B} \boldsymbol{y},
$$

with $\boldsymbol{x}, \boldsymbol{y} \in \mathcal{V}_{3}$ and $\boldsymbol{T} \in \mathfrak{L}\left(\mathcal{V}_{3}\right), \boldsymbol{A}, \boldsymbol{B} \in \mathfrak{L}\left(\mathcal{V}_{3}, S \cap \mathcal{G}\right)$. Therefore the inequality (7.14) becomes

$$
\begin{aligned}
& \mathbf{g} \cdot \tilde{\boldsymbol{K}}_{+} \boldsymbol{g}-\boldsymbol{E} \cdot\left(\tilde{\theta} \tilde{\boldsymbol{\sigma}}_{+}\right) \boldsymbol{E}+\boldsymbol{g} \cdot\left(\tilde{\boldsymbol{L}}-\tilde{\theta} \tilde{\boldsymbol{\tau}}^{T}\right) \boldsymbol{E}+\boldsymbol{g} \cdot\left[\tilde{\theta}\left(\partial_{\boldsymbol{g}} \tilde{\boldsymbol{f}}\right)_{\tilde{s}}\left(\partial_{\boldsymbol{E}} \tilde{\boldsymbol{\alpha}}_{\mathrm{em}}\right)_{\tilde{p}}^{z}\right] \boldsymbol{E}+ \\
& +\boldsymbol{E} \cdot\left[\tilde{\theta}\left(\partial_{\boldsymbol{E}} \tilde{\boldsymbol{f}}\right)_{\tilde{s}}\left(\partial_{\boldsymbol{E}} \tilde{\boldsymbol{\alpha}}_{\mathrm{em}}\right)_{\tilde{p}}^{p}\right] \boldsymbol{E} \leqslant 0 \quad \forall \boldsymbol{g}, \boldsymbol{E} \in \mathcal{V}_{3},
\end{aligned}
$$

whence

$$
\begin{aligned}
\boldsymbol{g} \cdot \tilde{\boldsymbol{K}}_{+} \boldsymbol{g}-\boldsymbol{E} \cdot\left\{\tilde{\theta}\left[\tilde{\boldsymbol{\alpha}}-\left(\partial_{\boldsymbol{E}} \tilde{\boldsymbol{f}}\right)_{\tilde{s}}\left(\partial_{\boldsymbol{E}} \tilde{\boldsymbol{\alpha}}_{\mathrm{em}}\right)^{x}\right]_{+}\right\} \boldsymbol{E}+ \\
+\boldsymbol{g} \cdot\left\{\tilde{\boldsymbol{L}}-\tilde{\theta}\left[\tilde{\boldsymbol{\tau}}-\left(\partial_{\boldsymbol{E}} \tilde{\alpha}_{\mathrm{em}}\right)_{\tilde{p}}\left(\partial_{\boldsymbol{g}} \tilde{\boldsymbol{f}}\right)_{\tilde{s}}^{T}\right]^{T}\right\} \boldsymbol{E} \leqslant 0 \quad \forall \boldsymbol{g}, \boldsymbol{E} \in \mathcal{V}_{\mathbf{3}} .
\end{aligned}
$$

If we set

$$
\tilde{\sigma}^{*}=\tilde{\sigma}-\left(\partial_{\boldsymbol{E}} \tilde{f}\right)_{\tilde{s}}\left(\partial_{\boldsymbol{E}} \tilde{\alpha}_{\mathrm{em}}\right)_{\tilde{p}}^{p}
$$

and

$$
\tilde{\tau}^{*}=\tilde{\tau}-\left(\partial_{E} \tilde{\alpha}_{\mathrm{em}}\right)_{\tilde{p}}\left(\partial_{g} \tilde{f}\right)_{\tilde{s}}^{t}
$$


as the "modified electric conductivity and the "modified" electrothermal conductivity (in a classical equilibrium state) respectively, we can write the last inequality $(7.16)$ in the more compact form

$$
\boldsymbol{g} \cdot \widetilde{\boldsymbol{K}}_{+} \boldsymbol{g}-\boldsymbol{E} \cdot\left(\tilde{\theta} \tilde{\boldsymbol{\sigma}}_{+}^{*}\right) \boldsymbol{E}+\boldsymbol{g} \cdot\left[\tilde{\boldsymbol{L}}-\tilde{\theta}\left(\tilde{\boldsymbol{\tau}}^{*}\right)^{T}\right] \boldsymbol{E} \leqslant 0
$$

for each $g$ and $\boldsymbol{E}$ in $\mathcal{V}_{3}$.

A further formal simplification can be achieved with the introduction of the "thermoelectric vector" $\boldsymbol{z} \equiv(\boldsymbol{g}, \boldsymbol{E}) \in \mathcal{V}_{6}$ and of the following symmetric tensor $\tilde{\boldsymbol{Z}} \in \mathfrak{L}\left(\mathfrak{V}_{6}\right)$, i.e.

$$
\tilde{\boldsymbol{Z}} \equiv\left(\frac{\tilde{\boldsymbol{K}}_{+}}{\frac{1}{2}\left[\boldsymbol{L}^{T}-\tilde{\theta} \tilde{\boldsymbol{\tau}}^{*}\right]} \mid \frac{\frac{1}{2}\left[\tilde{\boldsymbol{L}}-\tilde{\theta}\left(\tilde{\boldsymbol{\tau}}^{*}\right)^{T}\right]}{-\tilde{\theta} \tilde{\sigma}_{+}^{*}}\right), \quad \text { with } \tilde{\boldsymbol{Z}}^{T}=\tilde{\boldsymbol{Z}} .
$$

Thus (7.19) and (7.20) allow us to state the result:

THEORem 9. - The characteristic condition for the expansions $(7.8)_{1,2}$ to satisfy the chemical dissipation inequality for thermal and electric conduction "near" a classical equilibrium state of the mixture, is that the symmetric tensor (7.20) be negative semidefinite; i.e.

$$
z \cdot \tilde{Z} z \leqslant 0 \quad \forall z \in \mathcal{V}_{6}
$$

It is quite obvious that the above theorem has some interesting consequences.

Corollary 1. - A necessary condition for the expansions (7.8) $)_{1,2}$ to satisfy the chemical dissipation inequality for thermal and electric conduction "near" a classical equilibrium state of the mixture, is that the thermal conductivity tensor in the classical equilibrium state be negative semidefinite i.e.

$$
\boldsymbol{g} \cdot \tilde{\boldsymbol{K}} \boldsymbol{g} \leqslant 0 \quad \forall \boldsymbol{g} \in \mathcal{U}_{3} .
$$

Conollary 2. - A necessary condition for the expansions $(7.8)_{1,2}$ to satisfy the chemical dissipation inequality for thermal and electric conduction "near" a classical equilibrium state of the mixture, is that the "modified " electric conductivity tensor in the classical equilibrium state be either positive semidefinite $(\tilde{\theta}>0)$ or negative semidefinite $(\theta<0)$; i.e.

$$
\begin{array}{lll}
\text { if } \tilde{\theta}>0 & \boldsymbol{E} \cdot \tilde{\boldsymbol{\sigma}}^{*} \boldsymbol{E} \geqslant 0 & \forall \boldsymbol{E} \in \mathcal{V}_{3}, \\
\text { if } \tilde{\theta}<0 & \boldsymbol{E} \cdot \tilde{\sigma}^{*} \boldsymbol{E} \leqslant 0 & \forall \boldsymbol{E} \in \mathcal{V}_{3} .
\end{array}
$$

REMARK 1. - It is of some interest to point out that the necessary condition (7.22) on $\widetilde{\boldsymbol{K}}$ is exactly the same as the one which holds in a simple stationary rigid material 
with instantaneous electromagnetic response [24], [26]. On the contrary the conditions (7.24) on $\tilde{\sigma}^{*}$ represents an actual generalization with respect to the corresponding ones relative to a simple stationary rigid material with instantaneous electromagnetic response; in fact, for the mixture in exam, (7.23) imply restrictions not on the electric conductivity as in a simple material, but on the "modified " electric conductivity given by (7.17).

REMARK 2. - When the whole mixture can be regarded as isotropic "near" a state of classical equilibrium, the four tensors (7.7) reduce to

$$
\tilde{\boldsymbol{K}}=\tilde{K} \boldsymbol{I}, \quad \tilde{\boldsymbol{L}}=\tilde{L} \boldsymbol{I} \quad \text { and } \quad \tilde{\tau}=\tilde{\tau} \boldsymbol{I}, \quad \tilde{\sigma}=\tilde{\sigma} \boldsymbol{I},
$$

where $I$ is the unit tensor.

In this case the expansions $(7.8)_{1,2}$ become

$$
\tilde{\boldsymbol{q}}=\tilde{K} \boldsymbol{g}+\tilde{L} \boldsymbol{E}+\mathbf{0}\left(|s-\tilde{s}|^{2}\right)
$$

and

$$
\tilde{\boldsymbol{j}}=\tilde{\tau} \boldsymbol{g}+\tilde{\boldsymbol{\sigma}} \boldsymbol{E}+\mathbf{0}\left(|s-\tilde{s}|^{2}\right)
$$

when $(\theta, \boldsymbol{H}) \in \tilde{J}$.

Now one can adopt a procedure similar to the one outlined in refs. [27], [28] for isotropic solids in order to examine the meaning of higher-order expansions for $\tilde{\boldsymbol{q}}$ and $\tilde{\boldsymbol{j}}$ and the consistent restrictions on the associated coefficients. In this sense it is possible to give an exhaustive description of thermal and electric conduction "near » a classical equilibrium state of an isotropie mixture and to discuss the possible influences on thermoelectric, thermomagnetic and galvanomagnetic phenomena due to the presence of chemical effects.

\section{8. - Non-conducting mixtures and the law of mass action.}

In this last section we confine our attention to those mixtures in which neither heat nor current flows $\left(^{1}\right)$.

Our purpose is now to describe the most meaningfull consequences of this a priori assumption and to show how the "chemical dissipation inequality for thermal and electric conduction " yields naturally to an expression of the well known "law of mass action ".

In fact, when

$$
\tilde{\boldsymbol{q}} \equiv \mathbf{0} \quad \text { and } \quad \tilde{j} \equiv \mathbf{0}
$$

(1) For instance, this is the case of a mixture made up with thermally insulating dielectric materials provided no "disperion effect" (both electric and magnetic) is taken into account.

22 - Annali di Matematica 
the inequality (5.13) reduces to the simple form

$$
\theta \tilde{\boldsymbol{\alpha}}_{\mathrm{ern}} * \tilde{\boldsymbol{f}} \leqslant 0
$$

and may be called the "chemical dissipation inequality".

If we recall now the constitutive relation $(4.16)_{7},(8.2)$ becomes

$$
\theta \tilde{\boldsymbol{\alpha}}_{\mathrm{em}} * \stackrel{+}{\boldsymbol{w}} \leqslant 0
$$

which, when expressed in the constituent space $R^{n}$, reads

$$
\theta \sum_{1}^{n} \tilde{\alpha}_{\mathrm{em}}^{a} \stackrel{+}{w}_{a} \leqslant 0
$$

By the theorem 3 of the third section and by the direct sum decomposition (3.22) of the restricted reaction space $S \cap \mathcal{C}$, we can write [6], [7], [16]:

$$
\stackrel{+}{\boldsymbol{w}}=\sum_{1}^{d} \stackrel{+}{\boldsymbol{w}}_{k}
$$

with $\stackrel{+}{\boldsymbol{w}}_{k} \in \mathfrak{T}_{k}, k=1,2, \ldots, d ; d \in[1, n-\sigma]$.

The chemical reaction $\mathfrak{T}_{k}$ in $(3.22)$ can be chosen in such a way that $\mathfrak{T}_{k}$ is the one-dimensional subspace generated by $\boldsymbol{i}^{k}$, i.e. $\mathfrak{T}_{k}=L\left\{\boldsymbol{i}^{k}\right\}$, where $\boldsymbol{i}^{k}$ is the corresponding vector of the reciprocal of the basis generating $\delta \cap \mathcal{G}$. Then we have

$$
{\stackrel{+}{\boldsymbol{w}_{k}}}_{k}=\stackrel{+}{w}_{k} \dot{\boldsymbol{i}}^{k} \quad k=1,2, \ldots, d,
$$

whence

$$
\dot{w}=\sum_{1}^{d} \stackrel{+}{w}_{k l} \dot{i}^{k}
$$

Obviously the relation between the components of the reaction vector in $R^{n}$ (see $(3.1)_{2}$ ) and the components of the same vector in $S \cap \mathcal{G}$ (see (8.7)) is given by

$$
\stackrel{+}{w}_{a}=\sum_{1}^{d} \lambda_{a}^{k} \stackrel{+}{w}_{k} \quad a=1,2, \ldots, n
$$

where $w_{a}=\stackrel{+}{w} * \boldsymbol{e}_{a}, \stackrel{+}{w}_{k}=\stackrel{+}{\boldsymbol{w}} * \boldsymbol{i}_{k}$ and $\lambda_{a}^{k}=\dot{\boldsymbol{i}}^{k} * \boldsymbol{e}_{a}$ are the elements of the transformation matrix between the two basis $\left\{\boldsymbol{e}_{a}: a=1,2, \ldots, n\right\}$ and $\left\{\boldsymbol{i}^{k}: k=1,2, \ldots, d\right\}$.

Thus, by eqs. (8.8), the inequality (8.4) yields

$$
\theta \sum_{1}^{d}\left(\sum_{1}^{n} \lambda_{a}^{k} \hat{\alpha}_{\mathrm{em}}^{a}\right) \stackrel{+}{w}_{k} \leqslant 0
$$


We may consider the l.h.s. of the above inequality as a function of the scalars $\stackrel{+}{w}_{k}$, which attains either its maximum value $(\theta>0)$ or its minimum value $(\theta<0)$ when $\stackrel{+}{w}_{k}=0, k=1,2, \ldots, d$. Therefore, in any case, a necessary condition for (8.9) to hold is the well known law of mass action

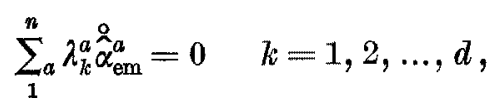

where the superscript " $\circ$ " denotes that the components of the electromagnetic chemical affinity must be evaluated for those states with null reaction vector $\stackrel{+}{w}$ (equilibrium states). Thus we arrive at the following main result:

THEOREM 10. - In a non-conducting mixture, a necessary condition for the validity of the ehemical dissipation inequality is given by the law of mass action.

It is of some interest to rewrite eqs. (8.10) in a more expressive form in order to illustrate the influence of the electromagnetic field, when linear isotropic constitutive relations for $\hat{\boldsymbol{D}}$ and $\hat{\boldsymbol{B}}$ are assumed. More precisely, eq. (5.19) gives

$$
\hat{\alpha}_{\mathrm{en}}^{a} \cong \hat{\alpha}_{0}^{a}-\left(\frac{\partial \hat{x}}{\partial w_{a}} E^{2}+\frac{\partial \hat{\mu}}{\partial w_{a}} H^{2}\right) \quad a=1,2, \ldots, n,
$$

and therefore eqs. (8.10) become

$$
\sum_{1}^{n} \lambda_{a}^{k}{\stackrel{\circ}{\hat{\alpha}_{0}^{a}}}^{a}-\left[\sum_{1}^{n} \lambda_{a}^{k}\left(\frac{\partial^{\circ} \hat{x}}{\partial w_{a}}\right) E^{2}+\sum_{1}^{n} \lambda_{a}^{k}\left(\frac{\partial \hat{\mu} \hat{\mu}}{\partial w_{a}}\right) H^{2}\right] \cong 0 \quad k=1,2, \ldots, a
$$

Setting

$$
\begin{aligned}
& \frac{1}{2} \stackrel{\circ}{\beta}^{k}=\sum_{1}^{n} \lambda_{a}^{k}\left(\frac{\partial \stackrel{\hat{x}}{\partial w_{a}}}{}\right) \quad k=1,2, \ldots, d \\
& \frac{1}{2} \hat{\gamma}^{k}=\sum_{1}^{n}{ }_{a} \lambda_{a}^{k}\left(\frac{\partial \circ}{\partial w_{a}}\right) \quad k=1,2, \ldots, d,
\end{aligned}
$$

we can write eqs. (8.11) in the form

$$
\sum_{1}^{n} a \lambda_{k}^{a} \stackrel{\alpha}{\alpha}_{0}^{a}-\frac{1}{2}\left(\hat{\hat{\beta}}^{k} E^{2}+\stackrel{\circ}{\hat{\gamma}}^{k} H^{2}\right) \cong 0 \quad k=1,2, \ldots, d,
$$

where the contribution of the electromagnetic field has been put in full relief [5, cap. XIV].

Both the general expression (8.10) and its special form (8.13) of the law of mass action yield as many restrictions as the number of the indipendent chemical reactions which can occur in the mixture. Obviously these restrictions must be satisfied 
by the indipendent variables in those states charaeterized by null reaction veetor, i.e. in equilibrium states.

REMARK. - Notice that, in many textbooks on Chemical Thermodynamies and Chemical Physics, the components of $\stackrel{\dot{w}}{w}$ with respect to the basis generating $\delta \cap \mathcal{G}$ are commonly denoted with $j_{k}$ and called reaction rates, namely $j_{k} \equiv \stackrel{+}{w}_{k} k=1,2, \ldots, d$. Moreover the components of $\hat{\alpha}_{\mathrm{em}}$ with respect to the basis $B^{-1}$ of $R^{n}$ are frequently denoted by $\hat{\mu}_{\mathrm{em}}^{a}$ and called electromagnetic chemical potentials, i.e. $\hat{\alpha}_{\mathrm{em}}^{a} \equiv \hat{\mu}_{\mathrm{em}}^{a} a=1,2, \ldots, n$.

Finally the transformation matrix between the base $B \equiv\left\{\boldsymbol{e}_{a}: a=1,2, \ldots, n\right\}$ and the base $\left\{i^{p}: p=1,2, \ldots, d\right\}$, i.e. the matrix $\left\|\lambda_{a}^{p}\right\|$, is sometimes denominated stoichiometric matrix.

Aknowledgement. - A warmfull thank to professor Ingo Müller of the John Hopkins University for some stimulating discussions.

\section{REFERENCES}

[1] C. A. Truesdell, Sulle basi della termomeccanica, I, Rend. Accad. Lincei, 22 (1957), pp. $33-38$.

[2] C. A. Truesdell, Sulle basi della termomeccanica, II, Rend. Accad. Lincei, 22 (1957), pp. 158.166.

[3] W. Noux, The axiomatic method with special reference to Geometry and Physics, Sect. 2, North-Holland Publ. Co. (1959).

[4] C. A. Trunspext - R. A. Touprs, The classical field theories, Handbuch der Physik, vol. III/1 (1960).

[5] J. K. Krnkwood - I. OpPenterm, Ohemical Thermodynamics, MeGraw Hill (1961).

[6] W. A. Gredb, Linear Algebra, 2nd ed., Springer (1963).

[7] G. D. Mostow - J. H. SAMPsore - J. MExeR, Fundamental structures of Algebra, Mc Graw Hill (1963).

[8] B. D. CoLEMAN - W. NoLL, The thermodynamics of elastic materials with heat conduction and viscosity, Arch. Rational Mech. Anal, 43 (1963), pp. 167-178.

[9] P. D. KrLLY, A reacting continuum, Int. J. Engng. Sei., 2 (1964), pp. 129-153.

[10] R. ArIs, Prolegomena to the rational analysis of systems of chemical reactions, Arch. Rational Mech. Anal., 19 (1965), pp. 81-99.

[11] B. D. CoLemar - M. E. GuRTw, Thermodynamics with internal state variables, Journ. Chem. Phys., 47 (1967), pp. 597-613.

[12 ]R. M. Bower, Towards a thermodynamics and mechanics of mixtures, Arch. Rational Mech. Anal., 24: (1967), pp. 370-403.

[13] C. A. TRUESDenL, Sulle basi della termodinamica delle miscele, Rend. Accad. Naz. Lincei, 44 (1968), pp. 381-383.

[14] R. M. Bowen, Thermochemistry of reacting materials, Journ. Chem. Phys., 49 (1968), pp. $1625-1637$.

[15] R. ARIS, Prolegomena to the rational analysis of systems of chemical reactions: some addenda, Arch. Rational Mech. Anal., 27 (1968), pp. 356-364.

[16] R. M. BowEN, On the stoichiometry of chemically reacting materials, Arch. Rational Mech. Anal., 29 (1968), pp. 114-124. 
[17] R. M. BowEx, Thermochemistry of reacting materials: Erratum, Journ. Chem. Phys., 50 (1969), pp. 4601-4602.

[18] C. A. Truespext, Rational thermodynamics, MoGraw Hill (1969).

[19] R. M. BoweN, The thermochemistry of a reacting mixture of elastio materials with diffusion, Arch. Rational Meoh. Anal., 34 (1969), pp. 97-127.

[20] R. M. BowEN - J. C. WIEsE, Diffusion in mixtures of elastic materials, Int. J. Engng. Sci., 7 (1969), pp. 689.722.

[21] R. M. BowEN - D. J. GARCIA, On the thermodynamics of mixtures with several temperatures, Int. J. Engng. Sci., 8 (1970), pp. 63-83.

[22] M. E. Gurtin - A. S. VARGas, On the classical theory of reacting fluid mixtures, Arch. Rational Meoh. Anal., 43 (1971), pp. 179-197.

[23] M. E. GuntrN, On the thermodynamics of chemically reacting fluid mixtures, Arch. Rational Mech. Anal., 43 (1971), pp. 199-212.

[24] B. D. Colminax - C. H. DILL, Thermodynamic restrictions on the constitutive equations of electromagnetic theory, Zeit. Angew. Math. Phys., 22 (1971), pp. 691-702.

[25] B. D. Coleman - E. H. DILL, On the thermodynamics of electromagnetic fields in materials with memory, Arch. Rational Mech. Anal., 41 (1971), pp. 132-162.

26] R. BoRghtesanI, The thermodynamics of rigid materials with "quasi electromagnetic" response, Meccanica (Journal of AIMETA), 9, no. 1 (1974).

[27] R. Borghesant - A. Morro, Thermodynamics and isotropy in thermal and electric conduction, Meccanica (Journal of AIMETA), 9, no. 2 (1974).

[28] R. BORGHESANI - A. MoRRo, Thermodynamic restrictions on thermoelectrie, themomagnetio and galvanomagnetic coefficients, Meceanica (Journal of AIMETA), 9, no. 3 (1974).

[29] R. BENACH - I. MulcER, Thermodynamics and the description of magnetizable, dielectric mixtures of fluids, Arch. Rational Mech. Anal., forteoming.

[30] R. BoRGHESANI, Mixtures of reacting rigid materials in the presence of electromagnetic fields, Pubbl. Ist. Mat. Un. Genova, II serie, no. 100 (1974). 